\title{
A MEASURE OF ASYMMETRY FOR MULTI-WAY CONTINGENCY TABLES WITH ORDERED CATEGORIES
}

\author{
Kouji Yamamoto* and Sadao Tomizawa ${ }^{\dagger}$
}

\begin{abstract}
For square contingency tables with ordered categories, Tomizawa, Miyamoto and Hatanaka (2001) considered a measure that represents the degree of departure from symmetry. This paper extends the measure to multi-way tables with ordered categories. The measure proposed is expressed by using the Cressie-Read power-divergence or the Patil-Taillie diversity index. The measure could be useful for comparing the degrees of departure from symmetry in several multi-way tables with ordered categories. Examples are given.
\end{abstract}

\section{Introduction}

Consider an $r \times r$ square contingency table with the same ordinal row and column classifications. Let $p_{i j}$ denote the probability that an observation will fall in the $i$-th row and $j$-th column of the table $(i=1, \ldots, r ; j=1, \ldots, r)$. The symmetry model is defined by

$$
p_{i j}=p_{j i} \quad(i=1, \ldots, r ; j=1, \ldots, r ; i \neq j) ;
$$

see, e.g., Bowker (1948) and Bishop, Fienberg and Holland (1975, p.282). The symmetry model may also be considered for multi-way $r^{T}(T \geq 3)$ contingency tables (see Bishop et al., 1975, p.300, and Agresti, 2002, p.440); see also Sections 2 and 3.

For square contingency tables, denote the row and column variables by $X_{1}$ and $X_{2}$, respectively, and let

$$
G_{i j}=\sum_{s=1}^{i} \sum_{t=j}^{r} p_{s t}=\mathrm{P}\left(X_{1} \leq i, X_{2} \geq j\right) \quad \text { when } \quad i<j,
$$

and

$$
G_{i j}=\sum_{s=i}^{r} \sum_{t=1}^{j} p_{s t}=\mathrm{P}\left(X_{1} \geq i, X_{2} \leq j\right) \quad \text { when } \quad i>j .
$$

Then the symmetry model can be expressed as

$$
G_{i j}=G_{j i} \quad(i=1, \ldots, r ; j=1, \ldots, r ; i \neq j) .
$$

\footnotetext{
* Department of Information Sciences, Faculty of Science and Technology, Tokyo University of Science, Noda City, Chiba 278-8510, Japan E-mail: yamamoto_kouji@ymail.plala.or.jp

${ }^{\dagger}$ Department of Information Sciences, Faculty of Science and Technology, Tokyo University of Science, Noda City, Chiba 278-8510, Japan E-mail: tomizawa@is.noda.tus.ac.jp

Key words: Kullback-Leibler information; Measure; Power-divergence; Shannon entropy; Symmetry
} 
When the symmetry model does not hold, we may be interested in whether other structure exists, such as extended symmetry (e.g., quasi-symmetry (Caussinus, 1965), or marginal homogeneity (Stuart, 1955); see also Bishop et al., 1975, Chapter 8) or for ordinal data some particular form of asymmetry (e.g., conditional symmetry (McCullagh, 1978), diagonalsparameter symmetry (Goodman, 1979), and so on; see also Agresti, 1984, p.202). When there is a structure of asymmetry in a square contingency table, we are also interested in measuring the degree of departure from symmetry. For square contingency tables with nominal categories, Tomizawa (1994) and Tomizawa, Seo and Yamamoto (1998) considered measures which represent the degree of departure from symmetry. For square contingency tables with ordered categories, Tomizawa, Miyamoto and Hatanaka (2001) considered a measure, $\phi^{(\lambda)}$, which represents the degree of departure from symmetry (see Appendix 1). For multi-way tables with nominal categories, Yamamoto (2004) considered a measure, $\Phi^{(\lambda)}$, to represent it (see Appendix 2), which includes the measures of Tomizawa (1994) and Tomizawa et al. (1998).

We can test goodness-of-fit of the symmetry in multi-way contingency tables using the test statistic, e.g., the likelihood ratio chi-squared statistic or Pearson's chi-squared statistic. However, the test statistics would not be suitable for comparing the degrees of asymmetry in several tables (see Section 7). Since the symmetry model for square tables with ordered categories can be expressed as (1), the measure $\phi^{(\lambda)}$ would be useful to see to what degree the cumulative probabilities $\left\{G_{i j}\right\}$ are distant from those with a symmetric structure. We are also interested in a measure that represents the degree of departure from symmetry for a multi-way table with ordered categories.

The purpose of this paper is (i) to give another expression of the symmetry model based on the cumulative probabilities (instead of cell probabilities) for multi-way contingency tables and (ii) to propose a measure, which represents the degree of departure from symmetry in multi-way contingency tables with ordered categories. Section 2 proposes the measure for the case of three-way contingency tables, and Section 3 extends it to $T$-way $(T \geq 3)$ tables. The measures proposed are useful for comparing the degrees of departure from symmetry. The proposed measure is an extension of the measure $\phi^{(\lambda)}$ (in Appendix 1) to the multi-way table.

\section{Case of three-way tables}

Consider an $r \times r \times r$ contingency table with ordered categories. Let $p_{i j k}$ denote the probability that an observation will fall in the $(i, j, k)$-th cell of the table for $1 \leq i, j, k \leq r$. Denote the $s$ th variable by $X_{s}(s=1,2,3)$.

\subsection{Symmetry model}

The symmetry $(S)$ model (Bishop et al., 1975, p.300) is defined by

$$
p_{i j k}=p_{i k j}=p_{j i k}=p_{j k i}=p_{k i j}=p_{k j i} \quad(1 \leq i, j, k \leq r) .
$$

Note that for a $3 \times 3 \times 3$ table, the $S$ model can be expressed as follows:

$$
\begin{gathered}
p_{123}=p_{132}=p_{213}=p_{231}=p_{312}=p_{321} \\
p_{112}=p_{121}=p_{211}, \quad p_{113}=p_{131}=p_{311}, \quad p_{223}=p_{232}=p_{322} \\
p_{122}=p_{212}=p_{221}, \quad p_{133}=p_{313}=p_{331}, \quad p_{233}=p_{323}=p_{332} .
\end{gathered}
$$


We shall consider another expression of the $S$ model based on the cumulative probabilities, which are defined in a similar way to the cumulative probabilities $\left\{G_{i j}\right\}$ in two-way tables. First, we shall define some sets about the cell's index. Let

$$
\begin{aligned}
& E_{1}=\{(i, j, k) \mid 1 \leq i<j<k \leq r\}, \\
& E_{2}=\{(i, j, k) \mid 1 \leq i=j<k \leq r\}, \\
& E_{3}=\{(i, j, k) \mid 1 \leq i<j=k \leq r\},
\end{aligned}
$$

and

$$
D(i, j, k)=\{(l, m, n) \mid(l, m, n) \text { is any permutation of }(i, j, k)\} .
$$

For example, when $r=3, E_{1}=\{(1,2,3)\}, E_{2}=\{(1,1,2),(1,1,3),(2,2,3)\}$ and $E_{3}=$ $\{(1,2,2),(1,3,3),(2,3,3)\}$.

Secondly, we shall consider the cumulative probability for each set $E_{t}(t=1,2,3)$ defined by, for $\left(k_{1}, k_{2}, k_{3}\right) \in D(1,2,3)$,

$$
\begin{aligned}
& G_{k_{1} k_{2} k_{3}(i, j, k)}^{(1)}=\mathrm{P}\left(X_{k_{1}} \leq i<X_{k_{2}} \leq j, k \leq X_{k_{3}}\right) \quad \text { for }(i, j, k) \in E_{1}, \\
& G_{k_{1} k_{2} k_{3}(i, j, k)}^{(2)}=\mathrm{P}\left(X_{k_{1}} \leq i, j \leq X_{k_{2}}<k \leq X_{k_{3}}\right) \quad \text { for }(i, j, k) \in E_{1}, \\
& G_{k_{1} k_{2} k_{3}(i, j, k)}^{(3)}=\mathrm{P}\left(X_{k_{1}}, X_{k_{2}} \leq j, k \leq X_{k_{3}}\right) \quad \text { for }(i, j, k) \in E_{2}, \\
& G_{k_{1} k_{2} k_{3}(i, j, k)}^{(4)}=\mathrm{P}\left(X_{k_{1}} \leq i, j \leq X_{k_{2}}, X_{k_{3}}\right) \quad \text { for }(i, j, k) \in E_{3} .
\end{aligned}
$$

Note that for $E_{1}$, we define the cumulative probabilities with two types. For example,

$$
\begin{aligned}
& G_{123(i, j, k)}^{(1)}=\mathrm{P}\left(X_{1} \leq i<X_{2} \leq j, k \leq X_{3}\right), \\
& G_{123(i, j, k)}^{(2)}=\mathrm{P}\left(X_{1} \leq i, j \leq X_{2}<k \leq X_{3}\right) .
\end{aligned}
$$

When $r=5$ and $(i, j, k)=(1,2,4)$,

$$
\begin{aligned}
G_{123(1,2,4)}^{(1)} & =\mathrm{P}\left(X_{1} \leq 1<X_{2} \leq 2,4 \leq X_{3}\right) \\
& =p_{124}+p_{125} \\
G_{123(1,2,4)}^{(2)} & =\mathrm{P}\left(X_{1} \leq 1,2 \leq X_{2}<4 \leq X_{3}\right) \\
& =p_{124}+p_{125}+p_{134}+p_{135} .
\end{aligned}
$$

When $r=5$ and $(i, j, k)=(2,2,4)$,

$$
\begin{aligned}
G_{123(2,2,4)}^{(3)} & =\mathrm{P}\left(X_{1}, X_{2} \leq 2,4 \leq X_{3}\right) \\
& =p_{114}+p_{124}+p_{214}+p_{224}+p_{115}+p_{125}+p_{215}+p_{225} .
\end{aligned}
$$

When $r=5$ and $(i, j, k)=(2,4,4)$,

$$
\begin{aligned}
G_{123(2,4,4)}^{(4)} & =\mathrm{P}\left(X_{1} \leq 2,4 \leq X_{2}, X_{3}\right) \\
& =p_{144}+p_{145}+p_{154}+p_{155}+p_{244}+p_{245}+p_{254}+p_{255} .
\end{aligned}
$$




\section{YAMAMOTO and TOMIZAWA}

Moreover, we consider the following four models based on the cumulative probabilities described above: the $S_{1}$ model is defined by

$$
G_{123(i, j, k)}^{(1)}=G_{k_{1} k_{2} k_{3}(i, j, k)}^{(1)} \quad \text { for }\left(k_{1}, k_{2}, k_{3}\right) \in D(1,2,3),(i, j, k) \in E_{1},
$$

the $S_{2}$ model is defined by

$$
G_{123(i, j, k)}^{(2)}=G_{k_{1} k_{2} k_{3}(i, j, k)}^{(2)} \quad \text { for }\left(k_{1}, k_{2}, k_{3}\right) \in D(1,2,3),(i, j, k) \in E_{1},
$$

the $S_{3}$ model is defined by

$$
G_{123(i, j, k)}^{(3)}=G_{k_{1} k_{2} k_{3}(i, j, k)}^{(3)} \quad \text { for }\left(k_{1}, k_{2}, k_{3}\right) \in D(1,2,3),(i, j, k) \in E_{2},
$$

and the $S_{4}$ model is defined by

$$
G_{123(i, j, k)}^{(4)}=G_{k_{1} k_{2} k_{3}(i, j, k)}^{(4)} \quad \text { for }\left(k_{1}, k_{2}, k_{3}\right) \in D(1,2,3),(i, j, k) \in E_{3} .
$$

Now we consider a $3 \times 3 \times 3$ table. If the $S_{1}, S_{2}, S_{3}$ and $S_{4}$ models hold, the following equations hold:

$$
\begin{gathered}
G_{123(1,2,3)}^{(1)}=G_{132(1,2,3)}^{(1)}=G_{213(1,2,3)}^{(1)}=G_{231(1,2,3)}^{(1)}=G_{312(1,2,3)}^{(1)}=G_{321(1,2,3)}^{(1)}, \\
G_{123(1,2,3)}^{(2)}=G_{132(1,2,3)}^{(2)}=G_{213(1,2,3)}^{(2)}=G_{231(1,2,3)}^{(2)}=G_{312(1,2,3)}^{(2)}=G_{321(1,2,3)}^{(2)}, \\
G_{123(1,1,3)}^{(3)}=G_{132(1,1,3)}^{(3)}=G_{231(1,1,3)}^{(3)}, \\
G_{123(1,1,2)}^{(3)}=G_{132(1,1,2)}^{(3)}=G_{231(1,1,2)}^{(3)}, \\
G_{123(2,2,3)}^{(3)}=G_{132(2,2,3)}^{(3)}=G_{231(2,2,3)}^{(3)}, \\
G_{123(1,3,3)}^{(4)}=G_{213(1,3,3)}^{(4)}=G_{312(1,3,3)}^{(4)}, \\
G_{123(1,2,2)}^{(4)}=G_{213(1,2,2)}^{(4)}=G_{312(1,2,2)}^{(4)} \\
G_{123(2,3,3)}^{(4)}=G_{213(2,3,3)}^{(4)}=G_{312(2,3,3)}^{(4)} .
\end{gathered}
$$

From (2) and (3), we can obtain

$$
p_{123}=p_{132}=p_{213}=p_{312}=p_{231}=p_{321} .
$$


[Note that when $r=3,(2)$ is identical to (3), however, when $r \geq 4$, (2) is different from (3).] Also, from (4) and (7), we can obtain

$$
p_{113}=p_{131}=p_{311} \text { and } p_{133}=p_{313}=p_{331} .
$$

Moreover, from (5) and (9), we can obtain

$$
p_{112}=p_{121}=p_{211} \text { and } p_{233}=p_{323}=p_{332} .
$$

Finally, from (6) and (8), we can obtain

$$
p_{223}=p_{232}=p_{322} \text { and } p_{122}=p_{212}=p_{221} .
$$

Therefore, in a $3 \times 3 \times 3$ table, the $S$ model holds if and only if the $S_{1}, S_{2}, S_{3}$ and $S_{4}$ models hold. Generally, in an $r \times r \times r$ table, the $S$ model holds if and only if the $S_{1}, S_{2}, S_{3}$ and $S_{4}$ models hold.

\subsection{A measure of symmetry}

First, we shall consider a measure to represent the degree of departure from each $S_{t}$ $(t=1,2,3,4)$. Secondly, from Section 2.1 , we consider a measure to represent the degree of departure from $S$ by considering the weighted average of the four measures which represent the degree of departure from $S_{1}, S_{2}, S_{3}$ and $S_{4}$. Let for $t=1,2$,

$$
\left.\delta_{t}=\sum_{\left(j_{1}, j_{2}, j_{3}\right) \in E_{1}} \sum_{\left(k_{1}, k_{2}, k_{3}\right) \in D(1,2,3)} G_{k_{1} k_{2} k_{3}\left(j_{1}, j_{2}, j_{3}\right)}^{(t)}\right),
$$

for $t=3$,

$$
\left.\delta_{t}=\sum_{\left(j_{1}, j_{2}, j_{3}\right) \in E_{2}} \sum_{\substack{\left(k_{1}, k_{2}, k_{3}\right) \in D(1,2,3) \\ k_{1}<k_{2}}} G_{k_{1} k_{2} k_{3}\left(j_{1}, j_{2}, j_{3}\right)}^{(t)}\right)
$$

and for $t=4$,

$$
\left.\delta_{t}=\sum_{\left(j_{1}, j_{2}, j_{3}\right) \in E_{3}} \sum_{\left(k_{1}, k_{2}, k_{3}\right) \in D(1,2,3)} \sum_{k_{2}<k_{3}} G_{k_{1} k_{2} k_{3}\left(j_{1}, j_{2}, j_{3}\right)}^{(t)}\right) .
$$

For example, for a $3 \times 3 \times 3$ table, we see

$$
\begin{gathered}
\delta_{1}=\delta_{2}=p_{123}+p_{132}+p_{213}+p_{312}+p_{231}+p_{321} \\
\delta_{3}=\left(p_{113}+p_{131}+p_{311}\right)+\left(p_{112}+p_{121}+p_{211}+p_{113}+p_{131}+p_{311}\right)+\left(p_{113}+p_{131}\right. \\
\left.+p_{311}+p_{223}+p_{232}+p_{322}+p_{123}+p_{132}+p_{213}+p_{312}+p_{231}+p_{321}\right)
\end{gathered}
$$

and

$$
\begin{aligned}
\delta_{4}=\left(p_{133}+p_{313}\right. & \left.+p_{331}\right)+\left(p_{233}+p_{323}+p_{332}+p_{133}+p_{313}+p_{331}\right)+\left(p_{122}+p_{212}\right. \\
& \left.+p_{221}+p_{133}+p_{313}+p_{331}+p_{123}+p_{132}+p_{213}+p_{312}+p_{231}+p_{321}\right) .
\end{aligned}
$$


Consider a measure to represent the degree of departure from $S_{t}(t=1,2,3,4)$ as follows:

$$
\Psi_{t}^{(\lambda)}=\frac{\lambda(\lambda+1)}{6^{\lambda}-1} I_{t}^{(\lambda)} \quad(t=1,2)
$$

and

$$
\Psi_{t}^{(\lambda)}=\frac{\lambda(\lambda+1)}{3^{\lambda}-1} I_{t}^{(\lambda)} \quad(t=3,4)
$$

where for $t=1,2$,

$$
\begin{aligned}
I_{t}^{(\lambda)}=\frac{1}{\lambda(\lambda+1)} \sum_{\left(j_{1}, j_{2}, j_{3}\right) \in E_{1}} \sum_{\left(k_{1}, k_{2}, k_{3}\right) \in D(1,2,3)} \sum_{k_{1} k_{2} k_{3}\left(j_{1}, j_{2}, j_{3}\right)}^{*} & \\
& \times\left\{\left(\frac{G_{k_{1} k_{2} k_{3}\left(j_{1}, j_{2}, j_{3}\right)}^{*(t)}}{G_{\left(j_{1}, j_{2}, j_{3}\right)}^{S[t]}}\right)^{\lambda}-1\right\}
\end{aligned}
$$

for $t=3$,

$$
\begin{aligned}
I_{t}^{(\lambda)}=\frac{1}{\lambda(\lambda+1)} \sum_{\left(j_{1}, j_{2}, j_{3}\right) \in E_{2}} \sum_{\substack{\left.k_{1}, k_{2}, k_{3}\right) \in D(1,2,3) \\
k_{1}<k_{2}}} G_{k_{1} k_{2} k_{3}\left(j_{1}, j_{2}, j_{3}\right)}^{*(t)} & \\
& \times\left\{\left(\frac{G_{k_{1} k_{2} k_{3}\left(j_{1}, j_{2}, j_{3}\right)}^{*(t)}}{G_{\left(j_{1}, j_{2}, j_{3}\right)}^{S[t]}}\right)^{\lambda}-1\right\},
\end{aligned}
$$

for $t=4$,

$$
\begin{aligned}
I_{t}^{(\lambda)}=\frac{1}{\lambda(\lambda+1)} \sum_{\left(j_{1}, j_{2}, j_{3}\right) \in E_{3}} \sum_{\substack{\left(k_{1}, k_{2}, k_{3}\right) \in D(1,2,3) \\
k_{2}<k_{3}}} G_{k_{1} k_{2} k_{3}\left(j_{1}, j_{2}, j_{3}\right)}^{*(t)} & \\
& \times\left\{\left(\frac{G_{k_{1} k_{2} k_{3}\left(j_{1}, j_{2}, j_{3}\right)}^{*(t)}}{G_{\left(j_{1}, j_{2}, j_{3}\right)}^{S[t]}}\right)^{\lambda}-1\right\}
\end{aligned}
$$

and

$$
\begin{gathered}
G_{k_{1} k_{2} k_{3}\left(j_{1}, j_{2}, j_{3}\right)}^{*(t)}=\frac{G_{k_{1} k_{2} k_{3}\left(j_{1}, j_{2}, j_{3}\right)}^{(t)}}{\delta_{t}} \quad(t=1,2,3,4), \\
G_{\left(j_{1}, j_{2}, j_{3}\right)}^{S[t]}=\frac{1}{6} \sum_{\left(k_{1}, k_{2}, k_{3}\right) \in D(1,2,3)} \sum_{k_{1} k_{2} k_{3}\left(j_{1}, j_{2}, j_{3}\right)} \sum^{*(t)} \quad(t=1,2,3,4),
\end{gathered}
$$

where the value at $\lambda=0$ is taken to be the continuous limit as $\lambda \rightarrow 0$. Therefore, consider the measure to represent the degree of departure from $S$ (i.e., from $S_{1}, S_{2}, S_{3}$ and $S_{4}$ holding simultaneously) as follows:

$$
\Psi_{S}^{(\lambda)}=\frac{\delta_{1} \Psi_{1}^{(\lambda)}+\delta_{2} \Psi_{2}^{(\lambda)}+\delta_{3} \Psi_{3}^{(\lambda)}+\delta_{4} \Psi_{4}^{(\lambda)}}{\delta_{1}+\delta_{2}+\delta_{3}+\delta_{4}} \text { for } \lambda>-1
$$


The measure $\Psi_{S}^{(\lambda)}$ would represent the weighted average of $\Psi_{1}^{(\lambda)}, \Psi_{2}^{(\lambda)}, \Psi_{3}^{(\lambda)}$ and $\Psi_{4}^{(\lambda)}$, which represent the degree of departure from $S_{1}, S_{2}, S_{3}$ and $S_{4}$, respectively. When $\lambda=0$, for $t=1,2$,

$$
\Psi_{t}^{(0)}=\frac{1}{\log 6} I_{t}^{(0)}
$$

for $t=3,4$,

$$
\Psi_{t}^{(0)}=\frac{1}{\log 3} I_{t}^{(0)}
$$

with for $t=1,2$,

$$
I_{t}^{(0)}=\sum_{\left(j_{1}, j_{2}, j_{3}\right) \in E_{1}} \sum_{\left(k_{1}, k_{2}, k_{3}\right) \in D(1,2,3)} \sum_{k_{1} k_{2} k_{3}\left(j_{1}, j_{2}, j_{3}\right)}^{*(t)} \log \left(\frac{G_{k_{1} k_{2} k_{3}\left(j_{1}, j_{2}, j_{3}\right)}^{*(t)}}{G_{\left(j_{1}, j_{2}, j_{3}\right)}^{S[t]}}\right),
$$

for $t=3$,

$$
I_{t}^{(0)}=\sum_{\left(j_{1}, j_{2}, j_{3}\right) \in E_{2}} \sum_{\substack{\left.k_{1}, k_{2}, k_{3}\right) \in D(1,2,3) \\ k_{1}<k_{2}}} G_{k_{1} k_{2} k_{3}\left(j_{1}, j_{2}, j_{3}\right)}^{*(t)} \log \left(\frac{G_{k_{1} k_{2} k_{3}\left(j_{1}, j_{2}, j_{3}\right)}^{*(t)}}{G_{\left(j_{1}, j_{2}, j_{3}\right)}^{S[t]}}\right),
$$

and for $t=4$,

$$
I_{t}^{(0)}=\sum_{\left(j_{1}, j_{2}, j_{3}\right) \in E_{3}} \sum_{\left(k_{1}, k_{2}, k_{3}\right) \in D(1,2,3)} \sum_{k_{2}<k_{3}} G_{k_{1} k_{2} k_{3}\left(j_{1}, j_{2}, j_{3}\right)}^{*(t)} \log \left(\frac{G_{k_{1} k_{2} k_{3}\left(j_{1}, j_{2}, j_{3}\right)}^{*(t)}}{G_{\left(j_{1}, j_{2}, j_{3}\right)}^{S S t]}}\right) .
$$

Note that $I_{t}^{(\lambda)}(t=1,2,3,4)$ is the power-divergence between $\left\{G_{k_{1} k_{2} k_{3}\left(j_{1}, j_{2}, j_{3}\right)}^{*(t)}\right\}$ and $\left\{G_{\left(j_{1}, j_{2}, j_{3}\right)}^{S[t]}\right\}$, and especially when $\lambda=0, I_{t}^{(0)}$ is the Kullback-Leibler information between them. For details of power-divergence, see Cressie and Read (1984) and Read and Cressie (1988, p.15). Note that the value of $\lambda$ should be chosen by the user.

Next, we shall consider another expression of measure $\Psi_{S}^{(\lambda)}$ below. For $\left(k_{1}, k_{2}, k_{3}\right) \in$ $D(1,2,3)$, let for $\left(j_{1}, j_{2}, j_{3}\right) \in E_{1}$,

$$
G_{k_{1} k_{2} k_{3}\left(j_{1}, j_{2}, j_{3}\right)}^{c(t)}=\frac{G_{k_{1} k_{2} k_{3}\left(j_{1}, j_{2}, j_{3}\right)}^{(t)}}{\sum_{\left(l_{1}, l_{2}, l_{3}\right) \in D(1,2,3)} G_{l_{1} l_{2} l_{3}\left(j_{1}, j_{2}, j_{3}\right)}^{(t)}} \quad(t=1,2)
$$

for $\left(j_{1}, j_{2}, j_{3}\right) \in E_{2}$,

$$
G_{k_{1} k_{2} k_{3}\left(j_{1}, j_{2}, j_{3}\right)}^{c(t)}=\frac{G_{k_{1} k_{2} k_{3}\left(j_{1}, j_{2}, j_{3}\right)}^{(t)}}{\sum_{\substack{\left(l_{1}, l_{2}, l_{3}\right) \in D(1,2,3) \\ l_{1}<l_{2}}} G_{l_{1} l_{2} l_{3}\left(j_{1}, j_{2}, j_{3}\right)}^{(t)}} \quad(t=3),
$$

and for $\left(j_{1}, j_{2}, j_{3}\right) \in E_{3}$,

$$
\begin{gathered}
G_{k_{1} k_{2} k_{3}\left(j_{1}, j_{2}, j_{3}\right)}^{c(t)}=\frac{G_{k_{1} k_{2} k_{3}\left(j_{1}, j_{2}, j_{3}\right)}^{(t)}}{\sum_{\substack{\left(l_{1}, l_{2}, l_{3}\right) \in D(1,2,3) \\
l_{2}<l_{3}}} G_{l_{1} l_{2} l_{3}\left(j_{1}, j_{2}, j_{3}\right)}^{(t)}} \quad(t=4) . \\
-45-
\end{gathered}
$$


For example, for a $3 \times 3 \times 3$ table, we see

$$
\begin{gathered}
G_{123(123)}^{c(1)}=\frac{G_{123(123)}^{(1)}}{G_{123(123)}^{(1)}+G_{132(123)}^{(1)}+G_{213(123)}^{(1)}+G_{312(123)}^{(1)}+G_{231(123)}^{(1)}+G_{321(123)}^{(1)}}, \\
G_{123(223)}^{c(3)}=\frac{G_{123(223)}^{(3)}}{G_{123(223)}^{(3)}+G_{132(223)}^{(3)}+G_{231(223)}^{(3)}}
\end{gathered}
$$

and

$$
G_{123(233)}^{c(4)}=\frac{G_{123(233)}^{(4)}}{G_{123(233)}^{(4)}+G_{213(233)}^{(4)}+G_{312(233)}^{(4)}} .
$$

Then the $\Psi_{t}^{(\lambda)}$ may be expressed as for $t=1,2$,

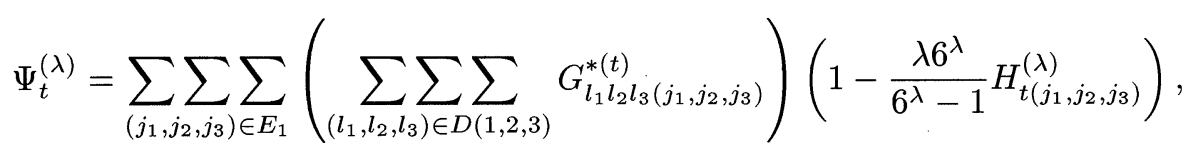

for $t=3$,

$$
\left.\Psi_{t}^{(\lambda)}=\sum_{\left(j_{1}, j_{2}, j_{3}\right) \in E_{2}} \sum_{\substack{\left(l_{1}, l_{2}, l_{3}\right) \in D(1,2,3) \\ l_{1}<l_{2}}} G_{l_{1} l_{2} l_{3}\left(j_{1}, j_{2}, j_{3}\right)}^{*(t)}\right)\left(1-\frac{\lambda 3^{\lambda}}{3^{\lambda}-1} H_{t\left(j_{1}, j_{2}, j_{3}\right)}^{(\lambda)}\right),
$$

and for $t=4$,

$$
\left.\Psi_{t}^{(\lambda)}=\sum_{\left(j_{1}, j_{2}, j_{3}\right) \in E_{3}} \sum_{\substack{\left(l_{1}, l_{2}, l_{3}\right) \in D(1,2,3) \\ l_{2}<l_{3}}} G_{l_{1} l_{2} l_{3}\left(j_{1}, j_{2}, j_{3}\right)}^{*(t)}\right)\left(1-\frac{\lambda 3^{\lambda}}{3^{\lambda}-1} H_{t\left(j_{1}, j_{2}, j_{3}\right)}^{(\lambda)}\right),
$$

where the value at $\lambda=0$ is taken to be the continuous limit as $\lambda \rightarrow 0$, and with for $t=1,2$,

$$
\begin{aligned}
& \left.H_{t\left(j_{1}, j_{2}, j_{3}\right)}^{(\lambda)}=\frac{1}{\lambda}\left\{1-\sum_{\left(k_{1}, k_{2}, k_{3}\right) \in D(1,2,3)} \sum_{k_{1} k_{2} k_{3}\left(j_{1}, j_{2}, j_{3}\right)}\right)^{\lambda+1}\right\}(\lambda \neq 0), \\
& H_{t\left(j_{1}, j_{2}, j_{3}\right)}^{(0)}=-\sum_{\left(k_{1}, k_{2}, k_{3}\right) \in D(1,2,3)}^{c(t)} \sum_{k_{1} k_{2} k_{3}\left(j_{1}, j_{2}, j_{3}\right)} \log \left(G_{k_{1} k_{2} k_{3}\left(j_{1}, j_{2}, j_{3}\right)}^{c(t)}\right),
\end{aligned}
$$

for $t=3$,

$$
\begin{aligned}
& H_{t\left(j_{1}, j_{2}, j_{3}\right)}^{(\lambda)}=\frac{1}{\lambda}\left\{1-\sum_{\substack{\left(k_{1}, k_{2}, k_{3}\right) \in D(1,2,3) \\
k_{1}<k_{2}}}\left(G_{k_{1} k_{2} k_{3}\left(j_{1}, j_{2}, j_{3}\right)}^{c(t)}\right)^{\lambda+1}\right\}(\lambda \neq 0), \\
& H_{t\left(j_{1}, j_{2}, j_{3}\right)}^{(0)}=-\sum_{\substack{\left(k_{1}, k_{2}, k_{3}\right) \in D(1,2,3) \\
k_{1}<k_{2}}} G_{k_{1} k_{2} k_{3}\left(j_{1}, j_{2}, j_{3}\right)}^{c(t)} \log \left(G_{k_{1} k_{2} k_{3}\left(j_{1}, j_{2}, j_{3}\right)}^{c(t)}\right),
\end{aligned}
$$


and for $t=4$,

$$
\begin{aligned}
H_{t\left(j_{1}, j_{2}, j_{3}\right)}^{(\lambda)} & =\frac{1}{\lambda}\left\{1-\sum_{\substack{\left(k_{1}, k_{2}, k_{3}\right) \in D(1,2,3) \\
k_{2}<k_{3}}}\left(G_{k_{1} k_{2} k_{3}\left(j_{1}, j_{2}, j_{3}\right)}^{c(t)}\right)^{\lambda+1}\right\} \quad(\lambda \neq 0), \\
H_{t\left(j_{1}, j_{2}, j_{3}\right)}^{(0)} & =-\sum_{\substack{\left(k_{1}, k_{2}, k_{3}\right) \in D(1,2,3) \\
k_{2}<k_{3}}} \sum_{k_{1} k_{2} k_{3}\left(j_{1}, j_{2}, j_{3}\right)}^{c(t)} \log \left(G_{k_{1} k_{2} k_{3}\left(j_{1}, j_{2}, j_{3}\right)}^{c(t)}\right) .
\end{aligned}
$$

Note that $H_{t\left(j_{1}, j_{2}, j_{3}\right)}^{(\lambda)}$ is the Patil and Taillie (1982) diversity index for $G_{k_{1} k_{2} k_{3}\left(j_{1}, j_{2}, j_{3}\right)}^{c(t)}$, $\left(k_{1}, k_{2}, k_{3}\right) \in D(1,2,3)$, including the Shannon entropy when $\lambda=0$. For a $3 \times 3 \times 3$ table, for example,

$$
\begin{aligned}
& H_{1(123)}^{(\lambda)}=\frac{1}{\lambda}\left[1-\left(G_{123(123)}^{c(1)}\right)^{\lambda+1}-\left(G_{132(123)}^{c(1)}\right)^{\lambda+1}-\left(G_{213(123)}^{c(1)}\right)^{\lambda+1}\right. \\
& \left.-\left(G_{312(123)}^{c(1)}\right)^{\lambda+1}-\left(G_{231(123)}^{c(1)}\right)^{\lambda+1}-\left(G_{321(123)}^{c(1)}\right)^{\lambda+1}\right] \quad(\lambda \neq 0), \\
& H_{1(123)}^{(0)}=-G_{123(123)}^{c(1)} \log G_{123(123)}^{c(1)}-G_{132(123)}^{c(1)} \log G_{132(123)}^{c(1)} \\
& -G_{213(123)}^{c(1)} \log G_{213(123)}^{c(1)}-G_{312(123)}^{c(1)} \log G_{312(123)}^{c(1)} \\
& -G_{231(123)}^{c(1)} \log G_{231(123)}^{c(1)}-G_{321(123)}^{c(1)} \log G_{321(123)}^{c(1)} . \\
& H_{3(223)}^{(\lambda)}=\frac{1}{\lambda}\left[1-\left(G_{123(223)}^{c(3)}\right)^{\lambda+1}-\left(G_{132(223)}^{c(3)}\right)^{\lambda+1}-\left(G_{231(223)}^{c(3)}\right)^{\lambda+1}\right] \quad(\lambda \neq 0), \\
& H_{3(223)}^{(0)}=-G_{123(223)}^{c(3)} \log G_{123(223)}^{c(3)}-G_{132(223)}^{c(3)} \log G_{132(223)}^{c(3)}-G_{231(223)}^{c(3)} \log G_{231(223)}^{c(3)} \text {. }
\end{aligned}
$$

We note that

$$
0 \leq H_{t\left(j_{1}, j_{2}, j_{3}\right)}^{(\lambda)} \leq M^{(\lambda)}
$$

where

$$
\begin{aligned}
& M^{(\lambda)}=\frac{6^{\lambda}-1}{\lambda 6^{\lambda}} \text { for any }\left(j_{1}, j_{2}, j_{3}\right) \in E_{1} \quad \text { when } t=1,2, \\
& M^{(\lambda)}=\frac{3^{\lambda}-1}{\lambda 3^{\lambda}} \text { for any }\left(j_{1}, j_{2}, j_{3}\right) \in E_{t-1} \quad \text { when } t=3,4 .
\end{aligned}
$$

The $S$ model holds if and only if $H_{t\left(j_{1}, j_{2}, j_{3}\right)}^{(\lambda)}=M^{(\lambda)}$ for any $\left(j_{1}, j_{2}, j_{3}\right)$ and $t(t=1,2,3,4)$ and the degree of departure from $S$ is the largest if and only if $H_{t\left(j_{1}, j_{2}, j_{3}\right)}^{(\lambda)}=0$ for any $\left(j_{1}, j_{2}, j_{3}\right)$ and $t(t=1,2,3,4)$. When $\lambda=0$,

$$
0 \leq H_{t\left(j_{1}, j_{2}, j_{3}\right)}^{(0)} \leq M^{(0)}
$$

where

$$
\begin{aligned}
& M^{(0)}=\log 6 \text { for any }\left(j_{1}, j_{2}, j_{3}\right) \in E_{1} \quad \text { when } t=1,2, \\
& M^{(0)}=\log 3 \text { for any }\left(j_{1}, j_{2}, j_{3}\right) \in E_{t-1} \quad \text { when } t=3,4 .
\end{aligned}
$$


For a $3 \times 3 \times 3$ table, for example,

$$
\begin{aligned}
& 0 \leq H_{1(123)}^{(0)} \leq \log 6 \\
& 0 \leq H_{3(223)}^{(0)} \leq \log 3 .
\end{aligned}
$$

When the $S$ model holds, $G_{123(123)}^{c(1)}=G_{132(123)}^{c(1)}=G_{213(123)}^{c(1)}=G_{312(123)}^{c(1)}=G_{231(123)}^{c(1)}=$ $G_{321(123)}^{c(1)}=1 / 6$, and $G_{123(223)}^{c(3)}=G_{132(223)}^{c(3)}=G_{231(223)}^{c(3)}=1 / 3$, and so on; thus $H_{1(123)}^{(0)}=\log 6$ and $H_{3(223)}^{(0)}=\log 3$. When the degree of departure from $S$ is the largest, $H_{1(123)}^{(0)}=0$ and $H_{3(223)}^{(0)}=0$, and so on; namely, e.g., $G_{123(123)}^{c(1)}=1$ and $G_{132(123)}^{c(1)}=G_{213(123)}^{c(1)}=G_{312(123)}^{c(1)}=$ $G_{231(123)}^{c(1)}=G_{321(123)}^{c(1)}=0$, and $G_{123(223)}^{c(3)}=1$ and $G_{132(223)}^{c(3)}=G_{231(223)}^{c(3)}=0$, and so on.

Thus for $t=1,2,3,4, \Psi_{t}^{(\lambda)}$ must lie between 0 and 1 , namely, $\Psi_{S}^{(\lambda)}$ must lie between 0 and 1. We note that (i) $\Psi_{t}^{(\lambda)}=0$ (every $t=1,2,3,4$ ), i.e., $\Psi_{S}^{(\lambda)}=0$ if and only if the $S$ model holds, and (ii) $\Psi_{t}^{(\lambda)}=1$ (every $t=1,2,3,4$ ), i.e., $\Psi_{S}^{(\lambda)}=1$ if and only if the degree of departure from $S$ is the largest in the sense that, for $t=1,2$ and $\left(j_{1}, j_{2}, j_{3}\right) \in E_{1}$,

$$
G_{k_{1} k_{2} k_{3}\left(j_{1}, j_{2}, j_{3}\right)}^{c(t)}=\left\{\begin{array}{l}
1 \text { for }\left(k_{1}, k_{2}, k_{3}\right)=\left(l_{0}, m_{0}, n_{0}\right) \\
\text { with one }\left(l_{0}, m_{0}, n_{0}\right) \in D(1,2,3), \\
0 \text { for otherwise }
\end{array}\right.
$$

namely,

$$
G_{l_{0} m_{0} n_{0}\left(j_{1}, j_{2}, j_{3}\right)}^{(t)} \neq 0 \text { and } G_{k_{1} k_{2} k_{3}\left(j_{1}, j_{2}, j_{3}\right)}^{(t)}=0 \text { for }\left(k_{1}, k_{2}, k_{3}\right) \neq\left(l_{0}, m_{0}, n_{0}\right)
$$

and it is defined in a similar way for $t=3,4$ and $\left(j_{1}, j_{2}, j_{3}\right) \in E_{t-1}$. For example, for a $3 \times 3 \times 3$ table, (i) $\Psi_{1}^{(\lambda)}=1$ indicates that one of $G_{123(123)}^{(1)}, G_{132(123)}^{(1)}, G_{213(123)}^{(1)}, G_{312(123)}^{(1)}$, $G_{231(123)}^{(1)}$ and $G_{321(123)}^{(1)}$ is not zero and the others are all zeros, and (ii) $\Psi_{3}^{(\lambda)}=1$ indicates that one of $G_{123(112)}^{(3)}, G_{132(112)}^{(3)}$ and $G_{231(112)}^{(3)}$ is not zero and the others are zeros, one of $G_{123(113)}^{(3)}, G_{132(113)}^{(3)}$ and $G_{231(113)}^{(3)}$ is not zero and the others are zeros, and one of $G_{123(223)}^{(3)}$, $G_{132(223)}^{(3)}$ and $G_{231(223)}^{(3)}$ is not zero and the others are zeros.

According to the Patil-Taillie diversity index or the power-divergence, the degree of departure from $S$ increases as the value of $\Psi_{S}^{(\lambda)}$ increases.

\section{Case of multi-way tables}

Consider an $r^{T}(T \geq 3)$ contingency table with ordered categories. Let $p_{i_{1} \ldots i_{T}}$ denote the probability that an observation will fall in the $\left(i_{1}, \ldots, i_{T}\right)$-th cell of the table $\left(i_{t}=\right.$ $1, \ldots, r ; t=1, \ldots, T)$. Denote the $s$ th variable by $X_{s}(s=1, \ldots, T)$.

\subsection{Symmetry model for multi-way tables}

The symmetry $(S)$ model (Agresti, 2002, p.440) is defined by

$$
p_{i_{1} \ldots i_{T}}=p_{j_{1} \ldots j_{T}}
$$

for $i_{t}=1, \ldots, r ; t=1, \ldots, T$, and $\left(j_{1}, \ldots, j_{T}\right) \in D\left(i_{1}, \ldots, i_{T}\right)$, where

$$
D\left(i_{1}, \ldots, i_{T}\right)=\left\{\left(j_{1}, \ldots, j_{T}\right) \mid\left(j_{1}, \ldots, j_{T}\right) \text { is any permutation of }\left(i_{1}, \ldots, i_{T}\right)\right\}
$$


For a fixed $s(s=2, \ldots, T)$, let

$$
\begin{aligned}
E\left(r_{1}, \ldots, r_{s}\right)=\left\{\left(j_{1}, \ldots, j_{T}\right) \mid 1 \leq j_{1} \leq \cdots \leq j_{T} \leq r\right. & \\
j_{1}=\cdots=j_{r_{1}}<j_{r_{1}+1}=\cdots & \left.=j_{r_{1}+r_{2}}<\cdots<j_{r_{1}+\cdots+r_{s-1}+1}=\cdots=j_{T}\right\}
\end{aligned}
$$

with

$$
r_{1}+\cdots+r_{s}=T \text { and } 1 \leq r_{i} \leq T-1 .
$$

For example, when $T=3$, for $s=3$,

$$
E(1,1,1)=\left\{\left(j_{1}, j_{2}, j_{3}\right) \mid 1 \leq j_{1}<j_{2}<j_{3} \leq r\right\},
$$

and for $s=2$,

$$
\begin{aligned}
& E(2,1)=\left\{\left(j_{1}, j_{2}, j_{3}\right) \mid 1 \leq j_{1}=j_{2}<j_{3} \leq r\right\}, \\
& E(1,2)=\left\{\left(j_{1}, j_{2}, j_{3}\right) \mid 1 \leq j_{1}<j_{2}=j_{3} \leq r\right\} .
\end{aligned}
$$

Note that $E(1,1,1), E(2,1)$ and $E(1,2)$ are identical to $E_{1}, E_{2}$ and $E_{3}$ defined in Section 2.1 , respectively. We shall consider an extension of the cumulative probabilities defined in Section 2.1 to multi-way tables.

For $\left(k_{1}, \ldots, k_{T}\right) \in D(1, \ldots, T)$, and a fixed $s(s=2, \ldots, T), l(l=1, \ldots, s-1)$, $\left(j_{1}, \ldots, j_{T}\right) \in E\left(r_{1}, \ldots, r_{s}\right)$, let

$$
\begin{gathered}
G_{k_{1} \ldots k_{T}\left(j_{1}, \ldots, j_{T}\right)}^{\left(r_{1}+\cdots+r_{l}\right)}=\mathrm{P}\left(X_{k_{1}}, \ldots, X_{k_{r_{1}}} \leq j_{r_{1}}<X_{k_{r_{1}+1}}, \ldots, X_{k_{r_{1}+r_{2}}} \leq j_{r_{1}+r_{2}}<\right. \\
X_{k_{r_{1}+r_{2}+1}, \ldots, X_{k_{r_{1}+r_{2}+r_{3}}}} \leq j_{r_{1}+r_{2}+r_{3}}<\ldots, X_{k_{r_{1}+\cdots+r_{l}}} \leq j_{r_{1}+\cdots+r_{l}}, \\
j_{r_{1}+\cdots+r_{l+1}} \leq X_{k_{r_{1}+\cdots+r_{l}+1}}, \ldots, X_{k_{r_{1}+\cdots+r_{l+1}}}<j_{r_{1}+\cdots+r_{l+2}} \leq \\
\left.X_{k_{r_{1}+\cdots+r_{l+1}+1}}, \cdots<j_{T} \leq X_{k_{r_{1}+\cdots+r_{s}+1}}, \ldots, X_{k_{T}}\right) .
\end{gathered}
$$

Note that the $\left(r_{1}+\cdots+r_{l}\right)$ variables $X_{k_{1}}, X_{k_{2}}, \ldots, X_{k_{r_{1}}+\cdots+r_{l}}$ are less than or equal to $j_{r_{1}+\cdots+r_{l}}$, and $T-\left(r_{1}+\cdots+r_{l}\right)$ variables $X_{k_{r_{1}+\cdots+r_{l}+1}, \ldots, X_{k_{T}} \text { are greater than or equal }}$ to $j_{r_{1}+\cdots+r_{l+1}}$. For example, when $T=3$, (i) for $s=3, l=2,\left(j_{1}, j_{2}, j_{3}\right) \in E(1,1,1)$,

$$
G_{k_{1} k_{2} k_{3}\left(j_{1}, j_{2}, j_{3}\right)}^{(2)}=\mathrm{P}\left(X_{k_{1}} \leq j_{1}<X_{k_{2}} \leq j_{2}, j_{3} \leq X_{k_{3}}\right),
$$

(ii) for $s=3, l=1,\left(j_{1}, j_{2}, j_{3}\right) \in E(1,1,1)$,

$$
G_{k_{1} k_{2} k_{3}\left(j_{1}, j_{2}, j_{3}\right)}^{(1)}=\mathrm{P}\left(X_{k_{1}} \leq j_{1}, j_{2} \leq X_{k_{2}}<j_{3} \leq X_{k_{3}}\right),
$$

(iii) for $s=2, l=1,\left(j_{1}, j_{2}, j_{3}\right) \in E(2,1)$,

$$
G_{k_{1} k_{2} k_{3}\left(j_{1}, j_{2}, j_{3}\right)}^{(2)}=\mathrm{P}\left(X_{k_{1}}, X_{k_{2}} \leq j_{2}, j_{3} \leq X_{k_{3}}\right)
$$

and (iv) for $s=2, l=1,\left(j_{1}, j_{2}, j_{3}\right) \in E(1,2)$,

$$
G_{k_{1} k_{2} k_{3}\left(j_{1}, j_{2}, j_{3}\right)}^{(1)}=\mathrm{P}\left(X_{k_{1}} \leq j_{1}, j_{2} \leq X_{k_{2}}, X_{k_{3}}\right) .
$$

Note that $(10),(11),(12)$ and (13) are identical to $G_{k_{1} k_{2} k_{3}\left(j_{1}, j_{2}, j_{3}\right)}^{(t)}(t=1,2,3,4)$ defined in Section 2.1, respectively. 
In a similar way to Section 2.1, we consider the following model: for fixed $s(s=$ $2, \ldots, T), l(l=1, \ldots, s-1)$ and $\left(j_{1}, \ldots, j_{T}\right) \in E\left(r_{1}, \ldots, r_{s}\right)$,

$$
G_{1 \ldots T\left(j_{1}, \ldots, j_{T}\right)}^{\left(r_{1}+\cdots+r_{l}\right)}=G_{k_{1} \ldots k_{T}\left(j_{1}, \ldots, j_{T}\right)}^{\left(r_{1}+\cdots+r_{l}\right)}
$$

where $\left(k_{1}, \ldots, k_{T}\right) \in D(1, \ldots, T)$. We shall refer to (14) as the $S_{\left(r_{1}, \ldots, r_{s}\right)}^{\left[r_{1}+\cdots+r_{l}\right]}$ model. Note that when $T=3, S_{(1,1,1)}^{[2]}, S_{(1,1,1)}^{[1]}, S_{(2,1)}^{[2]}$ and $S_{(1,2)}^{[1]}$ models are identical to $S_{1}, S_{2}, S_{3}$ and $S_{4}$ defined in Section 2.1, respectively. In a similar way to Section 2.1, we point out that the $S$ model holds if and only if any of the $S_{\left(r_{1}, \ldots, r_{s}\right)}^{\left[r_{1}+\cdots+r_{l}\right]}$ models hold.

\subsection{A measure of symmetry for multi-way tables}

We shall extend the measure proposed in Section 2.2 to multi-way tables. In a similar way to Section 2.2, we consider the measure to represent the degree of departure from $S$ by considering the weighted average of the measures, which represent the degree of departure from each $S_{\left(r_{1}, \ldots, r_{s}\right)}^{\left[r_{1}+\cdots+r_{l}\right]}$.

Let

$$
\begin{gathered}
\delta_{\left(r_{1}, \ldots, r_{s}\right)}^{\left[r_{1}+\cdots+r_{l}\right]}=\sum_{\left(j_{1}, \ldots, j_{T}\right) \in E\left(r_{1}, \ldots, r_{s}\right)}\left(\sum_{\left(k_{1}, \ldots, k_{T}\right) \in D^{*}\left(r_{1}, \ldots, r_{s}\right)} \sum_{k_{1} \ldots k_{T}\left(j_{1}, \ldots, j_{T}\right)}^{\left[r_{1}+\cdots+r_{l}\right]}\right), \\
\delta=\sum_{\left(r_{1}, \ldots, r_{s}\right) \in S^{*}} \sum_{l=1} \ldots \sum_{\left(r_{1}, \ldots, r_{s}\right)}\left(\sum_{l}^{s-1} \delta^{\left[r_{1}+\cdots+r_{l}\right]}\right), \\
D^{*}\left(r_{1}, \ldots, r_{s}\right)=\left\{\left(k_{1}, \ldots, k_{T}\right) \mid\left(k_{1}, \ldots, k_{T}\right) \text { is any permutation }(1, \ldots, T)\right. \text { with } \\
\left.k_{1}<\cdots<k_{r_{1}}, k_{r_{1}+1}<\cdots<k_{r_{1}+r_{2}}, \ldots, k_{r_{1}+\cdots+r_{s-1}}<\cdots<k_{T}\right\},
\end{gathered}
$$

and

$$
S^{*}=\left\{\left(r_{1}, \ldots, r_{s}\right) \mid s=2, \ldots, T, r_{1}+\cdots+r_{s}=T, 1 \leq r_{i} \leq T-1(i=1, \ldots, s)\right\} .
$$

Consider a measure to represent the degree of departure from $S_{\left(r_{1}, \ldots, r_{s}\right)}^{\left[r_{1}+\cdots+r_{l}\right]}$ model as follows: for $\left(r_{1}, \ldots, r_{s}\right) \in S^{*}$ and $l(l=1, \ldots, s-1)$,

$$
\Psi_{\left(r_{1}, \ldots, r_{s}\right)}^{\left[r_{1}+\cdots+r_{l}\right](\lambda)}=\frac{\lambda(\lambda+1)}{\left({ }_{T} \mathrm{C}_{r_{1} \ldots r_{s}}\right)^{\lambda}-1} I_{\left(r_{1}, \ldots, r_{s}\right)}^{\left[r_{1}+\cdots+r_{l}\right](\lambda)}
$$

where

$$
\begin{aligned}
& I_{\left(r_{1}, \ldots, r_{s}\right)}^{\left[r_{1}+\cdots+r_{l}\right](\lambda)}=\frac{1}{\lambda(\lambda+1)} \sum_{\left(j_{1}, \ldots, j_{T}\right) \in E\left(r_{1}, \ldots, r_{s}\right)} \sum_{\left(k_{1}, \ldots, k_{T}\right) \in D^{*}} \ldots \sum_{\left(r_{1}, \ldots, r_{s}\right)} G_{k_{1} \ldots k_{T}\left(j_{1}, \ldots, j_{T}\right)}^{*\left[r_{1}+\cdots+r_{l}\right]} \\
& \times\left\{\left(\frac{G_{k_{1} \ldots k_{T}\left(j_{1}, \ldots, j_{T}\right)}^{*\left[r_{1}+\cdots+r_{l}\right]}}{G_{\left(j_{1}, \ldots, j_{T}\right)}^{S\left[r_{1}+\cdots+r_{l}\right]}}\right)^{\lambda}-1\right\},
\end{aligned}
$$

with

$$
G_{k_{1} \ldots k_{T}\left(j_{1}, \ldots, j_{T}\right)}^{*\left[r_{1}+\ldots+r_{l}\right]}=\frac{G_{k_{1} \ldots k_{T}\left(j_{1}, \ldots, j_{T}\right)}^{\left[r_{1}+\ldots+r_{l}\right]}}{\delta_{\left(r_{1}, \ldots, r_{s}\right)}^{\left[r_{1}+\cdots+r_{l}\right]}}
$$




$$
\begin{gathered}
G_{\left(j_{1}, \ldots, j_{T}\right)}^{S\left[r_{1}+\cdots+r_{l}\right]}=\frac{1}{T !}\left(\sum_{\left(k_{1}, \ldots, k_{T}\right) \in D(1, \ldots, T)} \sum_{k_{1} \ldots k_{T}\left(j_{1}, \ldots, j_{T}\right)} \ldots G_{T}^{*\left[r_{1}+\cdots+r_{l}\right]}\right) \\
{ }_{T} \mathrm{C}_{r_{1} \ldots r_{s}}=\frac{T !}{r_{1} ! \ldots r_{s} !},
\end{gathered}
$$

and the value at $\lambda=0$ is taken to be the continuous limit as $\lambda \rightarrow 0$. Consider the measure to represent the degree of departure from $S$ (i.e., from $\left\{S_{\left(r_{1}, \ldots, r_{s}\right)}^{\left[r_{1}+\cdots+r_{l}\right]}\right\}$ models) as follows:

$$
\Psi_{S}^{(\lambda)}=\frac{1}{\delta} \sum_{\left(r_{1}, \ldots, r_{s}\right) \in S^{*}} \sum_{l=1}\left(\sum_{\left.l r_{1}, \ldots, r_{s}\right)}^{s-1} \delta_{\left(r_{1}, \ldots, r_{s}\right)}^{\left[r_{1}+\cdots+r_{l}\right]}\right) \quad \text { for } \lambda>-1
$$

Note that when $T=3$,

$$
\dot{\Psi}_{S}^{(\lambda)}=\frac{1}{\delta}\left(\delta_{(1,1,1)}^{[2]} \Psi_{(1,1,1)}^{[2](\lambda)}+\delta_{(1,1,1)}^{[1]} \Psi_{(1,1,1)}^{[1](\lambda)}+\delta_{(2,1)}^{[2]} \Psi_{(2,1)}^{[2](\lambda)}+\delta_{(1,2)}^{[1]} \Psi_{(1,2)}^{[1](\lambda)}\right)
$$

corresponds to $\Psi_{S}^{(\lambda)}$ defined in Section 2.2. When $\lambda=0, \Psi_{\left(r_{1}, \ldots, r_{s}\right)}^{\left[r_{1}+\cdots+r_{l}\right](\lambda)}$ is

$$
\begin{array}{r}
\Psi_{\left(r_{1}, \ldots, r_{s}\right)}^{\left[r_{1}+\cdots+r_{l}\right](0)}=\frac{1}{\log \left(\mathrm{C}_{\left.r_{1} \ldots r_{s}\right)}\right)} \sum_{\left(j_{1}, \ldots, j_{T}\right) \in E\left(r_{1}, \ldots, r_{s}\right)\left(k_{1}, \ldots, k_{T}\right) \in D^{*}\left(r_{1}, \ldots, r_{s}\right)} G_{k_{1} \ldots k_{T}\left(j_{1}, \ldots, j_{T}\right)}^{*\left[r_{1}+\cdots+r_{l}\right]} \\
\times \log \left(\frac{G_{k_{1} \ldots k_{T}\left(j_{1}, \ldots, j_{T}\right)}^{*\left[r_{1}+\cdots+r_{l}\right]}}{G_{\left(j_{1}, \ldots, j_{T}\right)}^{S\left[r_{1}+\cdots+r_{l}\right]}}\right) .
\end{array}
$$

Next, we shall consider another expression of measure. Let for $\left(j_{1}, \ldots, j_{T}\right) \in E\left(r_{1}, \ldots, r_{s}\right)$ and $\left(k_{1}, \ldots, k_{T}\right) \in D^{*}\left(r_{1}, \ldots, r_{s}\right)$,

$$
G_{k_{1} \ldots k_{T}\left(j_{1}, \ldots, j_{T}\right)}^{c\left[r_{1}+\cdots+r_{l}\right]}=\frac{G_{k_{1} \ldots k_{T}\left(j_{1}, \ldots, j_{T}\right)}^{\left[r_{1}+\cdots+r_{l}\right]}}{\sum_{\left(l_{1}, \ldots, l_{T}\right) \in D^{*}\left(r_{1}, \ldots, r_{s}\right)} \cdots G_{l_{1} \ldots l_{T}\left(j_{1}, \ldots, j_{T}\right)}^{\left[r_{1}+\cdots+r_{l}\right]}} .
$$

Then, for $\left(r_{1}, \ldots, r_{s}\right) \in S^{*}$, we also see

$$
\begin{aligned}
\left.\Psi_{\left(r_{1}, \ldots, r_{s}\right)}^{\left[r_{1}+\cdots+r_{l}\right](\lambda)}=\sum_{\left(j_{1}, \ldots, j_{T}\right) \in E\left(r_{1}, \ldots, r_{s}\right)} \sum_{\left(\sum_{1}, \ldots, l_{T}\right) \in D^{*}\left(r_{1}, \ldots, r_{s}\right)} \cdots G_{l_{1} \ldots l_{T}\left(j_{1}, \ldots, j_{T}\right)}^{*\left[r_{1}+\cdots+r_{l}\right]}\right) \\
\times\left[1-\frac{\lambda\left({ }_{T} \mathrm{C}_{r_{1} \ldots r_{s}}\right)^{\lambda}}{\left({ }_{T} \mathrm{C}_{r_{1} \ldots r_{s}}\right)^{\lambda}-1} H_{\left(j_{1}, \ldots, j_{T}\right)}^{\left[r_{1}+\cdots+r_{l}\right](\lambda)}\right],
\end{aligned}
$$

where

$$
H_{\left(j_{1}, \ldots, j_{T}\right)}^{\left[r_{1}+\cdots+r_{l}\right](\lambda)}=\frac{1}{\lambda}\left\{1-\sum_{\left(k_{1}, \ldots, k_{T}\right) \in D^{*}\left(r_{1}, \ldots, r_{s}\right)}\left(G_{k_{1} \ldots k_{T}\left(j_{1}, \ldots, j_{T}\right)}^{c\left[r_{1}+\cdots+r_{l}\right]}\right)^{\lambda+1}\right\}
$$

For $\left(r_{1}, \ldots, r_{s}\right) \in S^{*}, \Psi_{\left(r_{1}, \ldots, r_{s}\right)}^{\left[r_{1}+\cdots+r_{l}\right](\lambda)}(l=1, \ldots, s-1)$ must lie between 0 and 1 , and therefore $\Psi_{S}^{(\lambda)}$ must lie between 0 and 1. Also, (i) there is a structure of $S$ in the $r^{T}$ table if 
and only if $\Psi_{\left(r_{1}, \ldots, r_{s}\right)}^{\left[r_{1}+\cdots+r_{l}\right](\lambda)}=0$ for all $\left(r_{1}, \ldots, r_{s}\right) \in S^{*}, l=1, \ldots, s-1$, i.e., $\Psi_{S}^{(\lambda)}=0$, and (ii) the degree of departure from $S$ is the largest in the sense that, for $\left(j_{1}, \ldots, j_{T}\right) \in E\left(r_{1}, \ldots, r_{s}\right)$, $\left(r_{1}, \ldots, r_{s}\right) \in S^{*}, l=1, \ldots, s-1$, and $\left(k_{1}, \ldots, k_{T}\right) \in D^{*}\left(r_{1}, \ldots, r_{s}\right)$,

$$
G_{k_{1} \ldots k_{T}\left(j_{1}, \ldots, j_{T}\right)}^{c\left[r_{1}+\cdots+r_{l}\right]}=\left\{\begin{array}{l}
1 \text { for }\left(k_{1}, \ldots, k_{T}\right)=\left(k_{1}^{0}, \ldots, k_{T}^{0}\right) \\
\quad \text { with one }\left(k_{1}^{0}, \ldots, k_{T}^{0}\right) \in D^{*}\left(r_{1}, \ldots, r_{s}\right), \\
0 \text { for }\left(k_{1}, \ldots, k_{T}\right) \neq\left(k_{1}^{0}, \ldots, k_{T}^{0}\right),
\end{array}\right.
$$

if and only if $\Psi_{\left(r_{1}, \ldots, r_{s}\right)}^{\left[r_{1}+\cdots+r_{l}\right](\lambda)}=1$ for all $\left(r_{1}, \ldots, r_{s}\right) \in S^{*}, l=1, \ldots, s-1$, i.e., $\Psi_{S}^{(\lambda)}=1$.

\section{Approximate confidence interval for measure}

Let $n_{i_{1} \ldots i_{T}}$ denote the observed frequency in the $\left(i_{1}, \ldots, i_{T}\right)$-th cell of the $r^{T}$ table $\left(i_{t}=\right.$ $1, \ldots, r ; t=1, \ldots, T)$ with $T \geq 3$. Assuming that the $\left\{n_{i_{1} \ldots i_{T}}\right\}$ result from full multinomial sampling, we consider an approximate standard error and large-sample confidence interval for $\Psi_{S}^{(\lambda)}$, using the delta method, descriptions of which are given by Bishop et al. (1975, Sec.14.6) and Agresti (1984, p.185). The sample version of $\Psi_{S}^{(\lambda)}, \hat{\Psi}_{S}^{(\lambda)}$, is given by $\Psi_{S}^{(\lambda)}$ with $\left\{p_{i_{1} \ldots i_{T}}\right\}$ replaced by $\left\{\hat{p}_{i_{1} \ldots i_{T}}\right\}$, where $\hat{p}_{i_{1} \ldots i_{T}}=n_{i_{1} \ldots i_{T}} / n$ and $n=\sum \sum \cdots \sum n_{i_{1} \ldots i_{T}}$. Using the delta method, $\sqrt{n}\left(\hat{\Psi}_{S}^{(\grave{\lambda})}-\Psi_{S}^{(\lambda)}\right)$ has asymptotically $($ as $n \rightarrow \infty)$ a normal distribution with mean zero and variance $\sigma^{2}\left[\Psi_{S}^{(\lambda)}\right]$. See Appendix 3 for the expression for $\sigma^{2}\left[\Psi_{S}^{(\lambda)}\right]$.

Let $\hat{\sigma}^{2}\left[\Psi_{S}^{(\lambda)}\right]$ denote $\sigma^{2}\left[\Psi_{S}^{(\lambda)}\right]$ with $\left\{p_{i_{1} \ldots i_{T}}\right\}$ replaced by $\left\{\hat{p}_{i_{1} \ldots i_{T}}\right\}$. Then $\hat{\sigma}\left[\Psi_{S}^{(\lambda)}\right] / \sqrt{n}$ is an estimated standard error of $\hat{\Psi}_{S}^{(\lambda)}$, and $\hat{\Psi}_{S}^{(\lambda)} \pm z_{p / 2} \hat{\sigma}\left[\Psi_{S}^{(\lambda)}\right] / \sqrt{n}$ is an approximate $100(1-p) \%$ confidence interval for $\Psi_{S}^{(\lambda)}$, where $z_{p / 2}$ is the percentage point from the standard normal distribution corresponding to a two-tail probability equal to $p$.

\section{Comparison between measures}

We shall compare our measure $\Psi_{S}^{(\lambda)}$ with $\Phi^{(\lambda)}$ which is considered by Yamamoto (2004) (see Appendix 2 for $\Phi^{(\lambda)}$ ).

First, consider the artificial data in Table 1a, and the modified data in Table $1 \mathrm{~b}$ which are obtained by interchanging categories 1 and 2 . We see from Table $1 \mathrm{c}$ that (i) for each $\lambda$, the value of $\hat{\Psi}_{S}^{(\lambda)}$ for Table 1a is different from that for Table 1b, but (ii) for each $\lambda$, the value of $\hat{\Phi}^{(\lambda)}$ for Table 1a is equal to that for Table 1b. Generally, (i) the measure $\Psi_{S}^{(\lambda)}$ is not invariant under arbitrary similar permutations of row and column categories except the reverse order, but (ii) the measure $\Phi^{(\lambda)}$ is invariant under them. Thus $\Psi_{S}^{(\lambda)}$ should be used for the data on an ordinal scale and $\Phi^{(\lambda)}$ should be used for the data on a nominal scale.

Secondly, we shall compare the cases of $\Psi_{S}^{(\lambda)}=1$ and $\Phi^{(\lambda)}=1$ in three-way tables. As shown in Section $2.2, \Psi_{S}^{(\lambda)}=1$ indicates that the degree of departure from $S$ is the largest. On the other hand, $\Phi^{(\lambda)}=1$ indicates that, for $(l, m, n) \in D(i, j, k), i, j, k=1, \ldots, r$, $t=1 ; 2$,

$$
p_{l m n[t]}^{c}=\left\{\begin{array}{l}
1 \text { for }(l, m, n)=\left(l_{0}, m_{0}, n_{0}\right) \\
\quad \text { with a cell }\left(l_{0}, m_{0}, n_{0}\right) \in D(i, j, k) \\
0 \text { for }(l, m, n) \neq\left(l_{0}, m_{0}, n_{0}\right)
\end{array}\right.
$$

where

$$
p_{i j k[1]}^{c}=\frac{p_{i j k}}{p_{i j k}+p_{i k j}+p_{j i k}+p_{j k i}+p_{k i j}+p_{k j i}} \quad \text { for } \quad(i, j, k) \in E_{1}
$$


and

$$
p_{i j k[2]}^{c}=\frac{2 p_{i j k}}{p_{i j k}+p_{i k j}+p_{j i k}+p_{j k i}+p_{k i j}+p_{k j i}} \quad \text { for } \quad(i, j, k) \in E_{2} \text { or } E_{3} .
$$

Assuming that for each $(i, j, k)$ with $1 \leq i \leq j \leq k \leq r, \sum \sum \sum_{(l, m, n) \in D(i, j, k)} p_{l m n}>0$, $\Psi_{S}^{(\lambda)}=1$ implies $\Phi^{(\lambda)}=1$; however, $\Phi^{(\lambda)}=1$ does not always imply $\Psi_{S}^{(\lambda)}=1$. The definition of the maximum departure from $S$ (i.e. complete asymmetry) for the measure $\Psi_{S}^{(\lambda)}$, generally, depends on the ordering of the categories. However, the definition of complete asymmetry for the measure $\Phi^{(\lambda)}$ does not depend on the ordering of the categories. For example, consider the artificial data in Table $2 \mathrm{a}$, and the modified data in Table $2 \mathrm{~b}$ which are obtained by interchanging categories 2 and 3 . Then we see from Table $2 \mathrm{c}$ that (i) for each $\lambda$, the value of $\hat{\Psi}_{S}^{(\lambda)}$ for Table 2 a equals 1 (being the maximum value) but that for Table $2 \mathrm{~b}$ it is less than 1 , and (ii) for each $\lambda$, the value of $\hat{\Phi}^{(\lambda)}$ for Table $2 a$ equals 1 (being the maximum value) and it also equals 1 for Table $2 \mathrm{~b}$. These indicate that the definition of the maximum departure from $S$ for $\Psi_{S}^{(\lambda)}$ depends on the order of listing the categories, but for $\Phi^{(\lambda)}$ the definition does not depend on it.

When the measures are used for analyzing the data on an ordinal scale, $\Phi^{(\lambda)}$ does not use the information about the category ordering. Therefore, for the data on an ordinal scale, the measure $\Psi_{S}^{(\lambda)}$ rather than $\Phi^{(\lambda)}$ should be used when one wants to use the information about that ordering.

\section{Examples}

\subsection{Example 1}

Consider the data in Table 3 from the Meteorological Agency in Japan. These are obtained from the daily temperature at three places, Sapporo, Tokyo and Naha in Japan in 2001 and in 2005, using three levels, (1) below normals, (2) normals and (3) above normals. Table 4 gives the estimates of the measure $\Psi_{S}^{(\lambda)}$, the estimated approximate standard errors for $\hat{\Psi}_{S}^{(\lambda)}$ and the approximate $95 \%$ confidence intervals for $\Psi_{S}^{(\lambda)}$. Because the confidence intervals for $\Psi_{S}^{(\lambda)}$ applied to each of Tables $3 \mathrm{a}$ and $3 \mathrm{~b}$ do not include zero for any $\lambda(>-1)$ (see Table 4), these would indicate that there is not a structure of symmetry in each of Tables $3 \mathrm{a}$ and $3 \mathrm{~b}$.

In each of Tables $3 \mathrm{a}$ and $3 \mathrm{~b}$, the symmetry model indicates that the probability that the temperatures in (Sapporo, Tokyo, Naha) are $(1,2,3)$ equals the probability that they are $(1,3,2)$, the probability that they are $(2,1,3)$, the probability that they are $(3,1$, $2)$, the probability that they are $(2,3,1)$, and the probability that they are $(3,2,1)$, and so on. So, in such a sense, there is the structure of symmetry among the temperatures at Sapporo, Tokyo and Naha if the symmetry model holds; however, there is a difference among the temperatures at three points if the symmetry model does not hold. Now since there is not a structure of symmetry in each of Tables $3 \mathrm{a}$ and $3 \mathrm{~b}$, there is a difference between the temperatures at Sapporo, Tokyo and Naha in each of 2001 and 2005.

When the degrees of asymmetry in Tables $3 \mathrm{a}$ and $3 \mathrm{~b}$ are compared using the confidence interval for $\Psi_{S}^{(\lambda)}$, that is greater for Table 3 a than Table $3 b$. Therefore, we could estimate that the difference among the temperatures at three places in 2001 is greater than that in 2005.

Let $W^{(\lambda)}$ denote the power-divergence statistic for testing goodness-of-fit of the symmetry model for a three-way contingency table (see Appendix 4 for $W^{(\lambda)}$, and see Cressie 
Table 1: (a) Artificial data; (b) modified data obtained by interchanging categories 1 and 2 ; and (c) corresponding values of $\hat{\Psi}_{S}^{(\lambda)}$ and $\hat{\Phi}^{(\lambda)}$.

(a) Artificial data

\begin{tabular}{|c|c|c|c|c|c|c|c|c|c|}
\hline \multirow{3}{*}{$X_{1}$} & \multicolumn{3}{|c|}{ (1) } & \multicolumn{3}{|c|}{$\begin{array}{l}X_{3} \\
(2)\end{array}$} & \multicolumn{3}{|c|}{ (3) } \\
\hline & & $X_{2}$ & & & $X_{2}$ & & & $X_{2}$ & \\
\hline & (1) & $(2)$ & (3) & (1) & (2) & (3) & (1) & $(2)$ & (3) \\
\hline (1) & 83 & 52 & 37 & 77 & 15 & 32 & 52 & 44 & 39 \\
\hline (2) & 38 & 21 & 58 & 68 & 81 & 40 & 30 & 35 & 57 \\
\hline (3) & 59 & 43 & 10 & 25 & 29 & 17 & 40 & 82 & 70 \\
\hline
\end{tabular}

(b) Modified data

\begin{tabular}{|c|c|c|c|c|c|c|c|c|c|}
\hline \multirow{3}{*}{$X_{1}$} & \multicolumn{3}{|c|}{ (1) } & \multicolumn{3}{|c|}{$\begin{array}{l}X_{3} \\
(2) \\
\end{array}$} & \multicolumn{3}{|c|}{ (3) } \\
\hline & & $X_{2}$ & & & $X_{2}$ & & & $X_{2}$ & \\
\hline & (1) & (2) & (3) & (1) & (2) & (3) & (1) & $(2)$ & (3) \\
\hline (1) & 81 & 68 & 40 & 21 & 38 & 58 & 35 & 30 & 57 \\
\hline (2) & 15 & 77 & 32 & 52 & 83 & 37 & 44 & 52 & 39 \\
\hline (3) & 29 & 25 & 17 & 43 & 59 & 10 & 82 & 40 & 70 \\
\hline
\end{tabular}

(c) Values of $\hat{\Psi}_{S}^{(\lambda)}$ and $\hat{\Phi}^{(\lambda)}$

\begin{tabular}{cccccc}
\hline & \multicolumn{2}{c}{ For Table 1a } & & \multicolumn{2}{c}{ For Table 1b } \\
\cline { 2 - 3 } \cline { 5 - 6 }$\lambda$ & $\hat{\Psi}_{S}^{(\lambda)}$ & $\hat{\Phi}^{(\lambda)}$ & & $\hat{\Psi}_{S}^{(\lambda)}$ & $\hat{\Phi}^{(\lambda)}$ \\
\hline-0.4 & 0.023 & 0.053 & & 0.030 & 0.053 \\
0 & 0.028 & 0.067 & & 0.038 & 0.067 \\
0.6 & 0.028 & 0.072 & & 0.040 & 0.072 \\
1.0 & 0.026 & 0.069 & & 0.037 & 0.069 \\
1.6 & 0.021 & 0.059 & & 0.031 & 0.059 \\
\hline
\end{tabular}

and Read, 1984, and Read and Cressie, 1988, p.15, for details of the power-divergence test statistic). From the values of $W^{(\lambda)}$ in Table 5, we see that the symmetry model fits each of Tables $3 \mathrm{a}$ and $3 \mathrm{~b}$ very poorly.

\subsection{Example 2}

The data in Table 6 , taken directly from Bishop et al. $(1975$, p.305), give the $3 \times 3 \times 3$ array of counts of stationary two-step transitions in the panel survey of potential voters in Erie County, Ohio, 1940, which summarize the voting intentions of the 1940 presidential elections. Although the voter's supportive political party was classified into Republican, Democrat, and Undecided, we regard the voters with 'Undecided' as the middle class which could not decide Republican or Democrat, and give an order like Republican, Undecided, and Democrat.

Table 7 gives the estimates of the measure $\Psi_{S}^{(\lambda)}$, the estimated approximate standard errors for $\hat{\Psi}_{S}^{(\lambda)}$ and the approximate $95 \%$ confidence intervals for $\Psi_{S}^{(\lambda)}$. Because the confidence intervals for $\Psi_{S}^{(\lambda)}$ applied to the data in Table 6 do not include zero for any $\lambda(>-1)$ 
Table 2: (a) Artificial data; (b) modified data obtained by interchanging categories 2 and 3 ; and (c) the corresponding values of $\hat{\Psi}_{S}^{(\lambda)}$ and $\hat{\Phi}^{(\lambda)}$.

(a) Artificial data

\begin{tabular}{|c|c|c|c|c|c|c|c|c|c|}
\hline \multirow[b]{2}{*}{$X_{1}$} & \multicolumn{3}{|c|}{ (1) } & \multicolumn{3}{|c|}{$\begin{array}{l}X_{3} \\
(2)\end{array}$} & \multicolumn{3}{|c|}{ (3) } \\
\hline & (1) & $\begin{array}{l}X_{2} \\
(2)\end{array}$ & (3) & (1) & $\begin{array}{l}X_{2} \\
(2)\end{array}$ & (3) & (1) & $\begin{array}{l}X_{2} \\
(2)\end{array}$ & (3) \\
\hline (1) & 50 & 0 & 0 & 15 & 30 & 0 & 40 & 70 & 25 \\
\hline (2) & 0 & 0 & 0 & 0 & 45 & 0 & 0 & 20 & 35 \\
\hline (3) & 0 & 0 & 0 & 0 & 0 & 0 & 0 & 0 & 55 \\
\hline
\end{tabular}

(b) Modified data

\begin{tabular}{|c|c|c|c|c|c|c|c|c|c|}
\hline \multirow{3}{*}{$X_{1}$} & \multicolumn{3}{|c|}{ (1) } & \multicolumn{3}{|c|}{$X_{3}$} & \multicolumn{3}{|c|}{ (3) } \\
\hline & & $X_{2}$ & & & $X_{2}$ & & & $X_{2}$ & \\
\hline & (1) & $(2)$ & (3) & (1) & (2) & (3) & (1) & (2) & (3) \\
\hline (1) & 50 & 0 & 0 & 40 & 25 & 70 & 15 & 0 & 30 \\
\hline (2) & 0 & 0 & 0 & 0 & 55 & 0 & 0 & 0 & 0 \\
\hline (3) & 0 & 0 & 0 & 0 & 35 & 20 & 0 & 0 & 45 \\
\hline
\end{tabular}

(c) Values of $\hat{\Psi}_{S}^{(\lambda)}$ and $\hat{\Phi}^{(\lambda)}$

\begin{tabular}{cccccc}
\hline & \multicolumn{2}{c}{ For Table 2a } & & \multicolumn{2}{c}{ For Table 2b } \\
\cline { 2 - 3 } \cline { 5 - 6 }$\lambda$ & $\hat{\Psi}_{S}^{(\lambda)}$ & $\hat{\Phi}^{(\lambda)}$ & & $\hat{\Psi}_{S}^{(\lambda)}$ & $\hat{\Phi}^{(\lambda)}$ \\
\hline-0.4 & 1.0 & 1.0 & & 0.748 & 1.0 \\
0 & 1.0 & 1.0 & & 0.752 & 1.0 \\
0.6 & 1.0 & 1.0 & & 0.749 & 1.0 \\
1.0 & 1.0 & 1.0 & & 0.745 & 1.0 \\
1.6 & 1.0 & 1.0 & & 0.735 & 1.0 \\
\hline
\end{tabular}

(see Table 7), these would indicate that there is not a structure of symmetry in the data in Table 6.

For the data in Table 6, denote 'Republican', 'Undecided' and 'Democrat' by ' $R$ ', ' $U$ ' and ' $\mathrm{D}$ ', respectively. Let $\mathrm{P}(\mathrm{A}, \mathrm{B}, \mathrm{C})$ denote the probability that an individual has opinion $\mathrm{A}$ in time $t-2, \mathrm{~B}$ in time $t-1$, and $\mathrm{C}$ in time $t$. Then the symmetry model indicates that (i) $\mathrm{P}(\mathrm{R}, \mathrm{U}, \mathrm{D})=\mathrm{P}(\mathrm{R}, \mathrm{D}, \mathrm{U})=\mathrm{P}(\mathrm{U}, \mathrm{R}, \mathrm{D})=\mathrm{P}(\mathrm{D}, \mathrm{R}, \mathrm{U})=\mathrm{P}(\mathrm{U}, \mathrm{D}, \mathrm{R})=\mathrm{P}(\mathrm{D}, \mathrm{U}, \mathrm{R})$, (ii) $\mathrm{P}(\mathrm{R}, \mathrm{R}, \mathrm{D})=\mathrm{P}(\mathrm{R}, \mathrm{D}, \mathrm{R})=\mathrm{P}(\mathrm{D}, \mathrm{R}, \mathrm{R})$, (iii) $\mathrm{P}(\mathrm{D}, \mathrm{D}, \mathrm{R})=\mathrm{P}(\mathrm{D}, \mathrm{R}, \mathrm{D})=\mathrm{P}(\mathrm{R}, \mathrm{D}, \mathrm{D})$, and so on. Now since there is not a structure of symmetry in these data, we are interested in measuring to what degree the departure from symmetry is toward the maximum asymmetry by using the measure $\Psi_{S}^{(\lambda)}$. Note that the maximum asymmetry means that, e.g., (i) $\mathrm{P}(\mathrm{R}, \mathrm{U}, \mathrm{D}) \neq 0, \mathrm{P}(\mathrm{R}, \mathrm{D}, \mathrm{U})=\mathrm{P}(\mathrm{U}, \mathrm{R}, \mathrm{D})=\mathrm{P}(\mathrm{D}, \mathrm{R}, \mathrm{U})=\mathrm{P}(\mathrm{U}, \mathrm{D}, \mathrm{R})=\mathrm{P}(\mathrm{D}, \mathrm{U}, \mathrm{R})=0$, (ii) $\mathrm{P}(\mathrm{R}, \mathrm{R}, \mathrm{D}) \neq 0, \mathrm{P}(\mathrm{R}, \mathrm{D}, \mathrm{R})=\mathrm{P}(\mathrm{D}, \mathrm{R}, \mathrm{R})=0$, (iii) $\mathrm{P}(\mathrm{D}, \mathrm{D}, \mathrm{R}) \neq 0, \mathrm{P}(\mathrm{D}, \mathrm{R}, \mathrm{D})=$ $\mathrm{P}(\mathrm{R}, \mathrm{D}, \mathrm{D})=0$, and so on.

We shall investigate the degree of departure from symmetry in greater detail. For 
Table 3: The daily temperature at Sapporo, Tokyo and Naha in Japan in 2001 and 2005.

(a) in 2001

\begin{tabular}{|c|c|c|c|c|c|c|c|c|c|}
\hline \multirow{3}{*}{ at Sapporo } & \multicolumn{2}{|r|}{ (1) } & & & \multicolumn{2}{|c|}{$(2)$} & \multicolumn{3}{|c|}{ (3) } \\
\hline & $\mathrm{a}$ & Tol & & & Tok & & & Tok & \\
\hline & (1) & (2) & (3) & (1) & $(2)$ & $(3)$ & (1) & $(2)$ & (3) \\
\hline (1) & 17 & 6 & 1 & 17 & 19 & 12 & 18 & 25 & 8 \\
\hline (2) & 4 & 9 & 2 & 16 & 34 & 28 & 8 & 19 & 16 \\
\hline (3) & 0 & 3 & 3 & 3 & 25 & 39 & 1 & 8 & 24 \\
\hline
\end{tabular}

(b) in 2005

\begin{tabular}{|c|c|c|c|c|c|c|c|c|c|}
\hline \multirow{3}{*}{ at Sapporo } & \multicolumn{3}{|c|}{ (1) } & & $\begin{array}{l}\mathrm{Na} \\
(2)\end{array}$ & & \multicolumn{3}{|c|}{ (3) } \\
\hline & & Tol & & & Tok & & & Tok & \\
\hline & (1) & (2) & (3) & (1) & (2) & $(3)$ & (1) & (2) & (3) \\
\hline (1) & 15 & 9 & 1 & 25 & 22 & 6 & 6 & 7 & 8 \\
\hline$(2)$ & 11 & 10 & 4 & 24 & 37 & 18 & 2 & 23 & 10 \\
\hline (3) & 1 & 3 & 3 & 5 & 39 & 34 & 0 & 21 & 21 \\
\hline
\end{tabular}

Table 4: Estimates of $\Psi_{S}^{(\lambda)}$, estimated approximate standard errors for $\hat{\Psi}_{S}^{(\lambda)}$ and approximate $95 \%$ confidence intervals for $\Psi_{S}^{(\lambda)}$, applied to Table 3 .

\begin{tabular}{ccccc}
\hline Tables & Value of $\lambda$ & $\begin{array}{c}\text { Estimated } \\
\text { measure }\end{array}$ & $\begin{array}{c}\text { Standard } \\
\text { error }\end{array}$ & $\begin{array}{c}\text { Confidence } \\
\text { interval }\end{array}$ \\
\hline For Table 3a & -0.4 & 0.141 & 0.025 & $(0.092,0.189)$ \\
& 0 & 0.174 & 0.030 & $(0.116,0.233)$ \\
& 0.6 & 0.186 & 0.032 & $(0.124,0.249)$ \\
& 1.0 & 0.180 & 0.031 & $(0.118,0.241)$ \\
& 1.6 & 0.161 & 0.029 & $(0.103,0.218)$ \\
\hline For Table 3b & -0.4 & 0.051 & 0.013 & $(0.026,0.077)$ \\
& 0 & 0.064 & 0.017 & $(0.031,0.096)$ \\
& 0.6 & 0.069 & 0.019 & $(0.032,0.105)$ \\
& 1.0 & 0.067 & 0.019 & $(0.030,0.103)$ \\
& 1.6 & 0.059 & 0.017 & $(0.025,0.093)$ \\
\hline
\end{tabular}

instance, when $\lambda=0$, the estimated measure $\hat{\Psi}_{S}^{(0)}$ equals 0.189 (see Table 7 ). Thus for these data, the degree of departure from symmetry is estimated to be 18.9 percent of the maximum degree of departure from symmetry.

Table 8 gives the values of power-divergence statistic $W^{(\lambda)}$ for testing goodness-of-fit of the symmetry model. We see that the symmetry model fits the data in Table 6 very poorly. Note that we cannot see to what degree the departure from symmetry is toward the maximum asymmetry by using the test statistic $W^{(\lambda)}$. 
Table 5: Values of power-divergence statistic $W^{(\lambda)}$ (with 17 degrees of freedom) for testing goodness-of-fit of the symmetry model, applied to Tables $3 \mathrm{a}$ and $3 \mathrm{~b}$.

\begin{tabular}{ccc}
\hline$\lambda$ & For Table 3a & For Table 3b \\
\hline-0.4 & 128.83 & 65.33 \\
0 & 119.43 & 60.05 \\
0.6 & 118.71 & 57.69 \\
1.0 & 123.50 & 57.67 \\
1.6 & 137.97 & 59.20 \\
\hline
\end{tabular}

Table 6: Stationary two-step transitions in a panel study of potential voters in Erie County, Ohio, 1940.

\begin{tabular}{|c|c|c|c|c|c|c|c|c|c|}
\hline \multirow{3}{*}{ Time $t-2$} & & $\mathrm{R}$ & & \multicolumn{3}{|c|}{$\mathrm{U}$} & Time $t$ & D & \\
\hline & & $\mathrm{e} t$ & & & ne $t$ & & & $\mathrm{me}$ & -1 \\
\hline & $\mathrm{R}$ & $\mathrm{U}$ & D & $\mathrm{R}$ & $\mathrm{U}$ & $\mathrm{D}$ & $\mathrm{R}$ & $\mathrm{U}$ & $\mathrm{D}$ \\
\hline $\mathrm{R}$ & 557 & 17 & 3 & 16 & 21 & 0 & 6 & 5 & 8 \\
\hline $\mathrm{U}$ & 71 & 62 & 6 & 11 & 346 & 6 & 1 & 54 & 63 \\
\hline D & 18 & 4 & 9 & 5 & 24 & 22 & 0 & 10 & 435 \\
\hline
\end{tabular}

Taken directly from Bishop et al. (1975, p.305):

(R : Republican, U : Undecided, D : Democrat).

Table 7: Estimates of $\Psi_{S}^{(\lambda)}$, estimated approximate standard errors for $\hat{\Psi}_{S}^{(\lambda)}$ and approximate $95 \%$ confidence intervals for $\Psi_{S}^{(\lambda)}$, applied to Table 6 .

\begin{tabular}{cccc}
\hline Values of $\lambda$ & $\begin{array}{c}\text { Estimated } \\
\text { measure }\end{array}$ & $\begin{array}{c}\text { Standard } \\
\text { error }\end{array}$ & $\begin{array}{c}\text { Confidence } \\
\text { interval }\end{array}$ \\
\hline-0.4 & 0.153 & 0.018 & $(0.118,0.188)$ \\
0 & 0.189 & 0.022 & $(0.145,0.233)$ \\
0.6 & 0.203 & 0.024 & $(0.155,0.250)$ \\
1.0 & 0.196 & 0.024 & $(0.148,0.244)$ \\
1.6 & 0.175 & 0.023 & $(0.130,0.220)$ \\
\hline
\end{tabular}

\section{Concluding Remarks}

The measure $\hat{\Psi}_{S}^{(\lambda)}$ could be useful for measuring to what degree the departure from symmetry is toward the maximum asymmetry (described in Sections 2.2, 3.2 and 6.2) for ordinal data (although the test statistic $W^{(\lambda)}$ is not useful for measuring it).

The measure $\hat{\Psi}_{S}^{(\lambda)}$ could be useful for comparing the degrees of departure from symmetry in several tables with ordered categories. 


\section{YAMAMOTO and TOMIZAWA}

Table 8: Values of power-divergence statistic $W^{(\lambda)}$ (with 17 degrees of freedom) for testing goodness-of-fit of the symmetry model, applied to Table 6.

\begin{tabular}{cc}
\hline$\lambda$ & $W^{(\lambda)}$ \\
\hline-0.4 & 247.29 \\
0 & 229.76 \\
0.6 & 222.98 \\
1.0 & 225.55 \\
1.6 & 237.72 \\
\hline
\end{tabular}

For the measure $\Psi_{S}^{(\lambda)}$, the analyst may be interested in which value of $\lambda$ should be preferred. However, in comparing tables, it seems best to compare values of $\hat{\Psi}_{S}^{(\lambda)}$ for a range of values of $\lambda$. For example, consider the artificial data in Tables $9 \mathrm{a}$ and $9 \mathrm{~b}$. We see from Table $9 \mathrm{c}$ that the value of $\hat{\Psi}_{S}^{(0)}$ is greater for Table $9 \mathrm{a}$ than Table $9 \mathrm{~b}$, but the value of $\hat{\Psi}_{S}^{(1)}$ is less for Table $9 \mathrm{a}$ than Table $9 \mathrm{~b}$ (although the differences are slight in these cases). So, for these cases, it may be impossible to decide (by using $\hat{\Psi}_{S}^{(\lambda)}$ ) whether the degree of asymmetry is greater for Table $9 \mathrm{a}$ or Table $9 \mathrm{~b}$. But generally, for the comparison between two tables, it would be possible to draw a conclusion if $\Psi_{S}^{(\lambda)}$ (for every $\lambda$ ) is always greater (or always less) for one table than the other table.

Consider two tables, A and B. When one wants to compare the degrees of departure from symmetry, it may be dangerous to use the test statistic $W^{(\lambda)}$ because, for a fixed $\lambda$, the value of measure $\hat{\Psi}_{S}^{(\lambda)}$ may be less for Table A than Table B, but the value of $W^{(\lambda)}$ is greater for Table A than Table B. For example, consider the artificial data in Tables 10a and 10b. From Table 10c, it seems natural to conclude that the degree of departure from symmetry is greater for Table $10 \mathrm{a}$ than Table $10 \mathrm{~b}$. We see from Table 11 that the values of $\hat{\Psi}_{S}^{(\lambda)}$ are greater for Table 10a than Table 10b, but the values of $W^{(\lambda)}$ are less for Table 10a than Table 10b. Therefore, the measure $\hat{\Psi}_{S}^{(\lambda)}$ may be useful for comparing the degree of departure from symmetry in several multi-way tables.

The test statistic $W^{(\lambda)}$ does not depend on the order of listing the categories because it is invariant under arbitrary similar permutation of categories for each variable (although the measure $\Psi_{S}^{(\lambda)}$ is not invariant under them). Thus $W^{(\lambda)}$ is not suitable for measuring the degree of departure from symmetry for the data on an ordinal scale when one wants to use the information about the category ordering. It seems that the test statistic $W^{(\lambda)}$ should be used only for testing goodness-of-fit of symmetry.

\section{Acknowledgements}

The authors would like to thank two referees for very helpful comments. Please contact the authors about the way to calculate the estimated standard error for $\hat{\Psi}_{S}^{(\lambda)}$ and the confidence interval for $\Psi_{S}^{(\lambda)}$ in Examples.

\section{Appendix 1}

For an $r \times r$ table with ordered categories, the measure of departure from symmetry considered by Tomizawa et al. (2001) is given as follows: assuming that $\left\{G_{i j}+G_{j i}>0\right\}$ for 
Table 9: (a), (b) Artificial data ( $n$ is sample size) and (c) the corresponding values of $\hat{\Psi}_{S}^{(\lambda)}$.

(a) $n=1325$ (sample size)

\begin{tabular}{|c|c|c|c|c|c|c|c|c|c|}
\hline \multirow[b]{2}{*}{$X_{1}$} & & (1) & & \multicolumn{3}{|c|}{$\begin{array}{l}X_{3} \\
(2)\end{array}$} & \multicolumn{3}{|c|}{ (3) } \\
\hline & (1) & $\begin{array}{l}X_{2} \\
(2)\end{array}$ & (3) & (1) & $\begin{array}{l}X_{2} \\
(2)\end{array}$ & (3) & (1) & $\begin{array}{l}X_{2} \\
(2)\end{array}$ & (3) \\
\hline (1) & 95 & 80 & 50 & 45 & 90 & 40 & 70 & 15 & 15 \\
\hline (2) & 60 & 10 & 20 & 70 & 80 & 80 & 35 & 10 & 10 \\
\hline (3) & 50 & 55 & 30 & 40 & 55 & 35 & 60 & 40 & 85 \\
\hline
\end{tabular}

(b) $n=1220$

\begin{tabular}{|c|c|c|c|c|c|c|c|c|c|}
\hline \multirow{3}{*}{$X_{1}$} & \multicolumn{3}{|c|}{ (1) } & \multicolumn{3}{|c|}{$\begin{array}{l}X_{3} \\
(2)\end{array}$} & \multicolumn{3}{|c|}{ (3) } \\
\hline & & $X_{2}$ & & & $X_{2}$ & & & $X_{2}$ & \\
\hline & (1) & (2) & (3) & (1) & (2) & (3) & (1) & (2) & (3) \\
\hline (1) & 80 & 40 & 60 & 30 & 25 & 40 & 30 & 65 & 25 \\
\hline (2) & 35 & 20 & 50 & 65 & 40 & 65 & 55 & 55 & 45 \\
\hline (3) & 30 & 20 & 20 & 45 & 60 & 25 & 65 & 35 & 95 \\
\hline
\end{tabular}

(c) Values of $\hat{\Psi}_{S}^{(\lambda)}$

\begin{tabular}{ccc}
\hline$\lambda$ & For Table $9 \mathrm{a}$ & For Table $9 \mathrm{~b}$ \\
\hline-0.4 & 0.0271 & 0.0259 \\
0 & 0.0343 & 0.0336 \\
0.6 & $0.0357^{*}$ & 0.0362 \\
1.0 & $0.0332^{*}$ & 0.0344 \\
1.6 & $0.0274^{*}$ & 0.0294 \\
\hline
\end{tabular}

* indicates that $\hat{\Psi}_{S}^{(\lambda)}$ is less for Table 9a than for Table $9 \mathrm{~b}$.

$i \neq j$

$$
\phi^{(\lambda)}=\frac{\lambda(\lambda+1)}{2^{\lambda}-1} I^{(\lambda)} \text { for } \lambda>-1
$$

where $\Delta=\sum \sum_{s \neq t} G_{s t}, G_{i j}^{*}=G_{i j} / \Delta$ and $Q_{i j}^{*}=\left(G_{i j}^{*}+G_{j i}^{*}\right) / 2$,

$$
I^{(\lambda)}=\frac{1}{\lambda(\lambda+1)} \sum_{i=1}^{r} \sum_{\substack{j=1 \\ i \neq j}}^{r} G_{i j}^{*}\left\{\left(\frac{G_{i j}^{*}}{Q_{i j}^{*}}\right)^{\lambda}-1\right\},
$$

and the value at $\lambda=0$ is taken to be the continuous limit as $\lambda \rightarrow 0$. 
Table 10: Artificial $3 \times 3 \times 3$ data.

(a) $n=375$ (sample size)

\begin{tabular}{|c|c|c|c|c|c|c|c|c|c|}
\hline \multirow[b]{2}{*}{$X_{1}$} & \multicolumn{3}{|c|}{ (1) } & \multicolumn{3}{|c|}{$\begin{array}{l}X_{3} \\
(2)\end{array}$} & \multicolumn{3}{|c|}{ (3) } \\
\hline & (1) & $\begin{array}{l}X_{2} \\
(2)\end{array}$ & (3) & (1) & $\begin{array}{l}X_{2} \\
(2)\end{array}$ & (3) & (1) & $\begin{array}{l}X_{2} \\
(2)\end{array}$ & (3) \\
\hline (1) & 5 & 5 & 25 & 5 & 10 & 10 & 25 & 60 & 25 \\
\hline (2) & 5 & 15 & 10 & 35 & 5 & 15 & 10 & 10 & 30 \\
\hline (3) & 10 & 10 & 5 & 10 & 15 & 5 & 5 & 5 & 5 \\
\hline
\end{tabular}

(b) $n=1875$

\begin{tabular}{|c|c|c|c|c|c|c|c|c|c|}
\hline \multirow{3}{*}{$X_{1}$} & & (1) & & \multicolumn{3}{|c|}{$X_{3}$} & \multicolumn{3}{|c|}{ (3) } \\
\hline & & $X_{2}$ & & & $X_{2}$ & & & $X_{2}$ & \\
\hline & (1) & $(2)$ & (3) & (1) & (2) & (3) & (1) & (2) & (3) \\
\hline (1) & 25 & 25 & 125 & 25 & 50 & 50 & 125 & 200 & 125 \\
\hline (2) & 100 & 75 & 50 & 175 & 25 & 75 & 50 & 50 & 25 \\
\hline (3) & 50 & 50 & 25 & 50 & 75 & 100 & 25 & 100 & 25 \\
\hline
\end{tabular}

(c) the values of $\hat{G}_{k_{1} k_{2} k_{3}\left(j_{1}, j_{2}, j_{3}\right)}^{\left[r_{1}+\cdots+r_{l}\right]}$ for Tables $10 \mathrm{a}$ and $10 \mathrm{~b}$

\begin{tabular}{lccl}
\hline & $\left(j_{1}, j_{2}, j_{3}\right)$ & $r_{1}+\cdots+r_{l}$ & $\begin{array}{l}\hat{G}_{k_{1} k_{2} k_{3}\left(j_{1}, j_{2}, j_{3}\right)}^{\left[r_{1}+\cdots+r_{1}\right.} \\
\text { for various }\left(k_{1}, k_{2}, k_{3}\right) \in D(1,2,3)\end{array}$ \\
\hline For Table 10a & $(1,1,2)$ & 2 & $(0.08,0.08,0.04)$ \\
& $(1,1,3)$ & 2 & $(0.07,0.07,0.03)$ \\
& $(2,2,3)$ & 2 & $(0.28,0.16,0.12)$ \\
& $(1,2,3)$ & 1 & $(0.16,0.03,0.03,0.03,0.03,0.03)$ \\
& $(1,2,3)$ & 2 & $(0.16,0.03,0.03,0.03,0.03,0.03)$ \\
& $(1,2,2)$ & 1 & $(0.28,0.16,0.11)$ \\
& $(1,3,3)$ & 1 & $(0.07,0.01,0.01)$ \\
\hline For Table 10b & $(2,3,3)$ & 1 & $(0.15,0.03,0.03)$ \\
& $(1,1,2)$ & 2 & $(0.08,0.08,0.08)$ \\
& $(1,1,3)$ & 2 & $(0.07,0.07,0.03)$ \\
& $(2,2,3)$ & 2 & $(0.23,0.16,0.12)$ \\
& $(1,2,3)$ & 1 & $(0.11,0.03,0.03,0.03,0.03,0.03)$ \\
& $(1,2,3)$ & 2 & $(0.11,0.03,0.03,0.03,0.03,0.03)$ \\
& $(1,2,2)$ & 1 & $(0.23,0.16,0.11)$ \\
& $(1,3,3)$ & 1 & $(0.07,0.01,0.01)$ \\
$(2,3,3)$ & 1 & $(0.08,0.07,0.07)$ \\
\hline
\end{tabular}

\section{Appendix 2}

For an $r^{T}(T \geq 3)$ table with nominal categories, the measure of departure from symmetry considered by Yamamoto (2004) is given as follows:

$$
\begin{aligned}
& \Phi^{(\lambda)}=\frac{1}{\delta^{*}} \sum_{\left(r_{1}, \ldots, r_{T}\right) \in R^{*}} \sum_{r_{1} \ldots r_{T}} \Phi_{r_{1} \ldots r_{T}}^{(\lambda)} \quad \text { for } \quad \lambda>-1, \\
& -60-
\end{aligned}
$$


Table 11: Values of $\hat{\Psi}_{S}^{(\lambda)}$ and the power-divergence statistic $W^{(\lambda)}$ (with 17 degrees of freedom) for testing goodness-of-fit of the symmetry model, applied to Tables 10a and $10 \mathrm{~b}$.

\begin{tabular}{cccccc}
\hline & \multicolumn{2}{c}{ For Table 10a } & & \multicolumn{2}{c}{ For Table 10b } \\
\cline { 2 - 3 } \cline { 5 - 6 }$\lambda$ & $\hat{\Psi}_{S}^{(\lambda)}$ & $W^{(\lambda)}$ & & $\hat{\Psi}_{S}^{(\lambda)}$ & $W^{(\lambda)}$ \\
\hline-0.4 & 0.096 & 151.77 & & 0.045 & 549.13 \\
0 & 0.127 & 158.31 & & 0.059 & 557.08 \\
0.6 & 0.141 & 175.80 & & 0.064 & 587.03 \\
1.0 & 0.137 & 193.99 & & 0.061 & 620.54 \\
1.6 & 0.121 & 234.97 & & 0.052 & 695.65 \\
\hline
\end{tabular}

where

$$
\begin{gathered}
\delta_{r_{1} \ldots r_{T}}^{*}=\sum_{\left(i_{1}, \ldots, i_{T}\right) \in E_{\left(r_{1}, \ldots, r_{T}\right)}^{*}} p_{i_{1} \ldots i_{T}}, \\
\delta^{*}=\sum_{\left(r_{1}, \ldots, r_{T}\right) \in R^{*}} \ldots \sum_{r_{1} \ldots r_{T}}\left(=1-\sum_{k=1}^{r} p_{k \ldots k}\right), \\
E_{\left(r_{1}, \ldots, r_{T}\right)}^{*}=\left\{\left(i_{1}, \ldots, i_{T}\right) \mid i_{t}=1, \ldots, r ; t=1, \ldots, T, \text { where the } r_{1}, \mathrm{~s}\right. \\
\text { elements of }\left(i_{1}, \ldots, i_{T}\right) \text { are equal, } r_{2} \text { s elements are equal, } \\
\left.\ldots, \text { and } r_{T} \mathrm{~s} \text { elements are equal }\right\},
\end{gathered}
$$

for $\left(r_{1}, \ldots, r_{T}\right)$ which satisfies

$$
\begin{gathered}
r_{1}+\cdots+r_{T}=T \text { and } T-1 \geq r_{1} \geq \cdots \geq r_{T} \geq 0 \\
R^{*}=\left\{\left(r_{1}, \ldots, r_{T}\right) \mid r_{1}+\cdots+r_{T}=T, T-1 \geq r_{1} \geq \cdots \geq r_{T} \geq 0\right\}
\end{gathered}
$$

and the value at $\lambda=0$ is taken to be the limit as $\lambda \rightarrow 0$. For $\left(r_{1}, \ldots, r_{T}\right) \in R^{*}, \Phi_{r_{1} \ldots r_{T}}^{(\lambda)}$ in $\Phi^{(\lambda)}$ is defined by

$$
\Phi_{r_{1} \ldots r_{T}}^{(\lambda)}=\frac{\lambda(\lambda+1)}{\left({ }_{T} C_{r_{1} \ldots r_{T}}\right)^{\lambda}-1} I_{r_{1} \ldots r_{T}}^{(\lambda)},
$$

where

$$
\begin{gathered}
I_{r_{1} \ldots r_{T}}^{(\lambda)}=\frac{1}{\lambda(\lambda+1)} \sum_{\left(i_{1}, \ldots, i_{T}\right) \in E_{\left(r_{1}, \ldots, r_{T}\right)}^{*}} p_{i_{1} \ldots i_{T}\left[r_{1} \ldots r_{T}\right]}^{*}\left\{\left(\frac{p_{i_{1} \ldots i_{T}\left[r_{1} \ldots r_{T}\right]}^{*}}{p_{i_{1} \ldots i_{T}\left[r_{1} \ldots r_{T}\right]}^{s}}\right)^{\lambda}-1\right\} \\
p_{i_{1} \ldots i_{T}\left[r_{1} \ldots r_{T}\right]}^{*}=p_{i_{1} \ldots i_{T}} / \delta_{r_{1} \ldots r_{T}}^{*}, \\
p_{i_{1} \ldots i_{T}\left[r_{1} \ldots r_{T}\right]}^{s}=\sum_{\left(j_{1}, \ldots, j_{T}\right) \in D\left(i_{1}, \ldots, i_{T}\right)} p_{j_{1} \ldots j_{T}\left[r_{1} \ldots r_{T}\right]}^{*} /\left({ }_{T} C_{\left.r_{1} \ldots r_{T}\right)}\right) \\
{ }_{T} C_{r_{1} \ldots r_{T}}=\frac{T !}{r_{1} ! \ldots r_{T} !} .
\end{gathered}
$$




\section{Appendix 3}

Consider the $r^{T}$ table with $T \geq 3$. Using the delta method, $\sqrt{n}\left(\hat{\Psi}_{S}^{(\lambda)}-\Psi_{S}^{(\lambda)}\right)$ has asymptotically (as $n \rightarrow \infty$ ) a normal distribution with mean zero and variance $\sigma^{2}\left[\Psi_{S}^{(\lambda)}\right]$, where for $\lambda>-1(\lambda \neq 0)$,

$$
\left.\sigma^{2}\left[\Psi_{S}^{(\lambda)}\right]=\sum_{\operatorname{not}} \sum_{i_{1}=\cdots=i_{T}} \cdots \sum_{i_{1} \ldots i_{T}}\right)^{2} p_{i_{1} \ldots i_{T}}^{(\lambda)}
$$

where

$$
\begin{aligned}
& w_{i_{1} \ldots i_{T}}^{(\lambda)}=\frac{1}{\delta}\left(R_{i_{1} \ldots i_{T}}^{(\lambda)}-\Psi_{S}^{(\lambda)} V_{i_{1} \ldots i_{T}}\right) \\
& \left.R_{i_{1} \ldots i_{T}}^{(\lambda)}=\sum_{\left(r_{1}, \ldots, r_{s}\right) \in S^{*}} \sum_{l=1}^{s-1} \sum_{\left(r_{1}, \ldots, r_{s}\right)}^{\left[\left[r_{1}+\cdots+r_{l}\right]\right.} \Psi_{\left(r_{1}, \ldots, r_{s}\right)}^{\left[r_{1}+\cdots+r_{l}\right](\lambda)}+\delta_{\left(r_{1}, \ldots, r_{s}\right)}^{\left[r_{1}+\cdots+r_{l}\right]} \Psi_{\left(r_{1}, \ldots, r_{s}\right)}^{\left[\left[r_{1}+\cdots+r_{l}\right](\lambda)\right.}\right\} \\
& \left.V_{i_{1} \ldots i_{T}}=\sum_{\left(r_{1}, \ldots, r_{s}\right) \in S^{*}} \sum_{l=1}^{s-1} \sum_{\left(r_{1}, \ldots, r_{s}\right)}^{\prime\left[r_{1}+\cdots+r_{l}\right]}\right) \\
& \delta_{\left(r_{1}, \ldots, r_{s}\right)}^{\prime\left[r_{1}+\cdots+r_{l}\right]}=\sum_{\left(j_{1}, \ldots, j_{T}\right) \in E\left(r_{1}, \ldots, r_{s}\right)}\left(\sum_{\left(k_{1}, \ldots, k_{T}\right) \in D^{*}} \sum_{\left(r_{1}, \ldots, r_{s}\right)} \cdots I_{k_{1} \ldots k_{T}\left(j_{1}, \ldots, j_{T}\right)}^{\left[r_{1}+\cdots+r_{l}\right]}\right) \\
& I_{k_{1} \ldots k_{T}\left(j_{1}, \ldots, j_{T}\right)}^{\left[r_{1}+\cdots+r_{r}\right]}=I\left(i_{k_{1}}, \ldots, i_{k_{r_{1}}} \leq j_{r_{1}}<i_{k_{r_{1}+1}}, \ldots, i_{k_{r_{1}+r_{2}}} \leq j_{r_{1}+r_{2}}<\right. \\
& i_{k_{r_{1}+r_{2}+1}}, \ldots, i_{k_{r_{1}+r_{2}+r_{3}}} \leq j_{r_{1}+r_{2}+r_{3}}<\ldots, i_{k_{r_{1}+\cdots+r_{l}}} \leq j_{r_{1}+\cdots+r_{l}}, \\
& j_{r_{1}+\cdots+r_{l+1}} \leq i_{k_{r_{1}+\cdots+r_{l}+1}}, \ldots, i_{k_{r_{1}+\cdots+r_{l+1}}}<j_{r_{1}+\cdots+r_{l+2}} \leq i_{k_{r_{1}+\cdots+r_{l+1}+1}}, \\
& \left.\cdots<j_{T} \leq i_{k_{r_{1}+\cdots+r_{s-1}+1}}, \ldots, i_{k_{T}}\right),
\end{aligned}
$$

with

$$
I(A)=\left\{\begin{array}{l}
1 \text { when } A \text { holds } \\
0 \text { otherwise }
\end{array}\right.
$$

$$
\begin{aligned}
& \Psi_{\left(r_{1}, \ldots, r_{s}\right)}^{\prime\left[r_{1}+\cdots+r_{l}\right](\lambda)}=\frac{1}{\left({ }_{T} \mathrm{C}_{r_{1} \ldots r_{s}}\right)^{\lambda}-1} \\
& \times \sum_{\left(j_{1}, \ldots, j_{T}\right) \in E\left(r_{1}, \ldots, r_{s}\right)}\left\{\sum_{\left(k_{1}, \ldots, k_{T}\right) \in D^{*}\left(r_{1}, \ldots, r_{s}\right)} S_{k_{1} \ldots k_{T}\left(j_{1}, \ldots, j_{T}\right)}^{\left[r_{1}+\cdots+r_{l}\right]}\left(\frac{G_{k_{1} \ldots k_{T}\left(j_{1}, \ldots, j_{T}\right)}^{*\left[r_{1}+\cdots+r_{l}\right]}}{G_{\left(j_{1}, \ldots, j_{T}\right)}^{S\left[r_{1}+\cdots+r_{l}\right]}}\right)^{\lambda}\right. \\
& \left.+\lambda G_{k_{1} \ldots k_{T}\left(j_{1}, \ldots, j_{T}\right)}^{*\left[r_{1}+\cdots+r_{l}\right]}\left(\frac{G_{k_{1} \ldots k_{T}\left(j_{1}, \ldots, j_{T}\right)}^{*\left[r_{1}+\cdots+r_{l}\right]}}{G_{\left(j_{1}, \ldots, j_{T}\right)}^{S\left[r_{1}+\cdots+r_{l}\right]}}\right)^{\lambda-1} T_{k_{1} \ldots k_{T}\left(j_{1}, \ldots, j_{T}\right)}^{\left[r_{1}+\cdots+r_{l}\right]}\right\}
\end{aligned}
$$




$$
\begin{gathered}
S_{k_{1} \ldots k_{T}\left(j_{1}, \ldots, j_{T}\right)}^{\left[r_{1}+\cdots+r_{l}\right]}=\frac{I_{k_{1} \ldots k_{T}\left(j_{1}, \ldots, j_{T}\right)}^{\left[r_{1}+\cdots+r_{l}\right]} \delta_{\left(r_{1}, \ldots, r_{s}\right)}^{\left[r_{1}+\cdots+r_{l}\right]}-G_{k_{1} \ldots k_{T}\left(j_{1}, \ldots, j_{T}\right)}^{\left[r_{1}+\cdots+r_{l}\right]} \delta_{\left(r_{1}, \ldots, r_{s}\right)}^{\left[r_{1}+\cdots+r_{l}\right]}}{\left(\delta_{\left(r_{1}, \ldots, r_{s}\right)}^{\left[r_{1}+\cdots+r_{l}\right]}\right)^{2}} \\
T_{k_{1} \ldots k_{T}\left(j_{1}, \ldots, j_{T}\right)}^{\left[r_{1}+\cdots+r_{l}\right]}=\frac{S_{k_{1} \ldots k_{T}\left(j_{1}, \ldots, j_{T}\right)}^{\left[r_{1}+\cdots+r_{l}\right]} G_{\left(j_{1}, \ldots, j_{T}\right)}^{S\left[r_{1}+\cdots+r_{l}\right]}-G_{k_{1} \ldots k_{T}\left(j_{1}, \ldots, j_{T}\right)}^{*\left[r_{1}+\cdots+r_{l}\right]} U_{\left(j_{1}, \ldots, j_{T}\right)}^{\left[r_{1}+\cdots+r_{l}\right]}}{\left(G_{\left(j_{1}, \ldots, j_{T}\right)}^{S\left[r_{1}+\cdots+r_{l}\right]}\right)^{2}}, \\
U_{\left(j_{1}, \ldots, j_{T}\right)}^{\left[r_{1}+\cdots+r_{l}\right]}=\frac{1}{T !}\left(\sum_{\left(k_{1}, \ldots, k_{T}\right) \in D(1, \ldots, T)} \sum_{k_{1} \ldots k_{T}\left(j_{1}, \ldots, j_{T}\right)} \cdots \sum^{\left[r_{1}+\cdots+r_{l}\right]}\right)
\end{gathered}
$$

and for $\lambda=0$,

$$
\left.\sigma^{2}\left[\Psi_{S}^{(0)}\right]=\sum_{\text {not }} \sum_{i_{1}=\cdots=i_{T}} \cdots \sum_{i_{1} \ldots i_{T}}\right)^{2} p_{i_{1} \ldots i_{T}}
$$

where

$$
\begin{aligned}
& w_{i_{1} \ldots i_{T}}^{(0)}=\frac{1}{\delta}\left(R_{i_{1} \ldots i_{T}}^{(0)}-\Psi_{S}^{(0)} V_{i_{1} \ldots i_{T}}\right),
\end{aligned}
$$

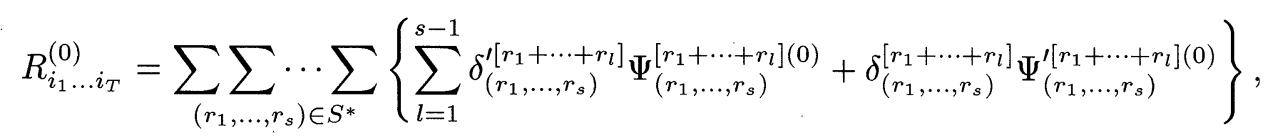

$$
\begin{aligned}
& \Psi_{\left(r_{1}, \ldots, r_{s}\right)}^{\prime\left[r_{1}+\cdots+r_{l}\right](0)}=\frac{1}{\log \left({ }_{T} \mathrm{C}_{r_{1} \ldots r_{s}}\right)} \\
& \times \sum_{\left(j_{1}, \ldots, j_{T}\right) \in E\left(r_{1}, \ldots, r_{s}\right)}\left\{\sum_{\left(k_{1}, \ldots, k_{T}\right) \in D^{*}\left(r_{1}, \ldots, r_{s}\right)} S_{k_{1} \ldots k_{T}\left(j_{1}, \ldots, j_{T}\right)}^{\left[r_{1}+\cdots+r_{l}\right]} \log \left(\frac{G_{k_{1} \ldots k_{T}\left(j_{1}, \ldots, j_{T}\right)}^{*\left[r_{1}+\cdots+r_{l}\right]}}{G_{\left(j_{1}, \ldots, j_{T}\right)}^{S\left[r_{1}+\cdots+r_{l}\right]}}\right)\right. \\
& \left.+G_{\left(j_{1}, \ldots, j_{T}\right)}^{S\left[r_{1}+\cdots+r_{l}\right]} T_{k_{1} \ldots k_{T}\left(j_{1}, \ldots, j_{T}\right)}^{\left[r_{1}+\cdots+r_{l}\right]}\right\} \text {. }
\end{aligned}
$$

Note that $\Psi_{\left(r_{1}, \ldots, r_{s}\right)}^{\prime\left[r_{1}+\cdots+r_{l}\right](0)}$ is equal to the limit of $\Psi_{\left(r_{1}, \ldots, r_{s}\right)}^{\prime\left[r_{1}+\cdots+r_{l}\right](\lambda)}$ as $\lambda \rightarrow 0$, namely, $\sigma^{2}\left[\Psi_{S}^{(0)}\right]$ is equal to the limit of $\sigma^{2}\left[\Psi_{S}^{(\lambda)}\right]$ as $\lambda \rightarrow 0$.

\section{Appendix 4}

For an $r \times r \times r$ table, the power-divergence chi-squared statistic for testing goodnessof-fit of the symmetry model is given by, for $-\infty<\lambda<\infty$,

$$
W^{(\lambda)}=\frac{2}{\lambda(\lambda+1)} \sum_{i=1}^{r} \sum_{j=1}^{r} \sum_{k=1}^{r} n_{i j k}\left[\left(\frac{6 n_{i j k}}{n_{i j k}+n_{i k j}+n_{j i k}+n_{j k i}+n_{k i j}+n_{k j i}}\right)^{\lambda}-1\right],
$$

and the values at $\lambda=-1$ and $\lambda=0$ are taken to be the limits as $\lambda \rightarrow-1$ and $\lambda \rightarrow 0$, respectively. The number of degrees of freedom is $r(r-1)(5 r+2) / 6$. 


\section{REFERENCES}

Agresti, A. (1984). Analysis of Ordinal Categorical Data. New York: Wiley.

Agresti, A. (2002). Categorical Data Analysis, 2nd edition. New York: Wiley.

Bishop, Y. M. M., Fienberg, S. E. and Holland, P. W. (1975). Discrete Multivariate Analysis: Theory and Practice. Cambridge, Massachusetts: The MIT Press.

Bowker, A. H. (1948). A test for symmetry in contingency tables. Journal of the American Statistical Association 43, 572-574.

Caussinus, H. (1965). Contribution à l'analyse statistique des tableaux de corrélation. Annales de la Faculté des Sciences de l'Université de Toulouse 29, 77-182.

Cressie, N. A. C. and Read, T. R. C. (1984). Multinomial goodness-of-fit tests. Journal of the Royal Statistical Society, Series B 46, 440-464.

Goodman, L. A. (1979). Multiplicative models for square contingency tables with ordered categories. Biometrika 66, 413-418.

McCullagh, P. (1978). A class of parametric models for the analysis of square contingency tables with ordered categories. Biometrika 65, 413-418.

Patil, G. P. and Taillie, C. (1982). Diversity as a concept and its measurement. Journal of the American Statistical Association 77, 548-561.

Read, T. R. C. and Cressie, N. A. C. (1988). Goodness-of-Fit Statistics for Discrete Multivariate Data. New York: Springer.

Stuart, A. (1955). A test for homogeneity of the marginal distributions in a two-way classification. Biometrika 42, 412-416.

Tomizawa, S. (1994). Two kinds of measures of departure from symmetry in square contingency tables having nominal categories. Statistica Sinica 4, 325-334.

Tomizawa, S., Miyamoto, N. and Hatanaka, Y. (2001). Measure of asymmetry for square contingency tables having ordered categories. Australian and New Zealand Journal of Statistics 43, 335-349.

Tomizawa, S., Seo, T. and Yamamoto, H. (1998). Power-divergence-type measure of departure from symmetry for square contingency tables that have nominal categories. Journal of Applied Statistics 25, 387-398.

Yamamoto, H. (2004). A measure of departure from symmetry for multi-way contingency tables with nominal categories. Japanese Journal of Biometrics 25, 69-88.

(Received: June 15, 2007, Accepted: December 4, 2007) 